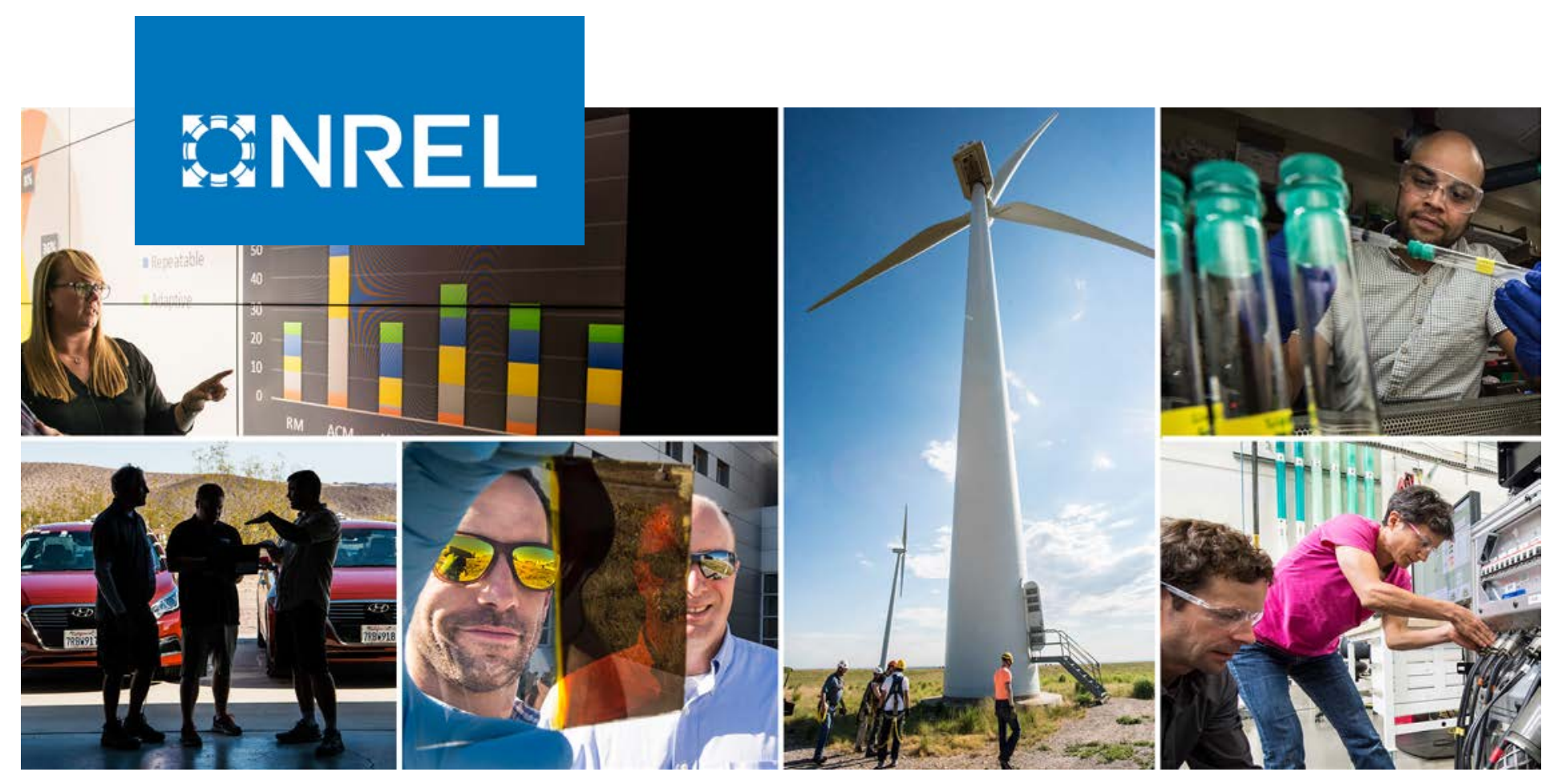

\title{
Low-Cost Multiparameter Device for Solar Resource Applications
}

Aron Habte, ${ }^{1}$ Afshin Andreas, ${ }^{1}$ Manajit Sengupta, ${ }^{1}$ Ranganath Narasappa, ${ }^{1}$ Andy Hoke, ${ }^{1}$ Peter Gotseff, ${ }^{1}$ Ramanathan Thiagarajan, ${ }^{1}$ Adam Wolf, ${ }^{2}$ Lorelí Carranza, ${ }^{2}$ and Danielle Watts ${ }^{2}$

${ }^{1}$ National Renewable Energy Laboratory

${ }^{2}$ Arable Labs, Inc.

NREL is a national laboratory of the U.S. Department of Energy Office of Energy Efficiency \& Renewable Energy

Operated by the Alliance for Sustainable Energy, LLC

This report is available at no cost from the National Renewable Energy Laboratory (NREL) at www.nrel.gov/publications.

\section{Technical Report}

NREL/TP-5D00-73104

March 2019 


\section{BNREL}

\section{Low-Cost Multiparameter Device for Solar Resource Applications}

Aron Habte, ${ }^{1}$ Afshin Andreas, ${ }^{1}$ Manajit Sengupta, ${ }^{1}$ Ranganath Narasappa, ${ }^{1}$ Andy Hoke, ${ }^{1}$ Peter Gotseff, ${ }^{1}$ Ramanathan Thiagarajan, ${ }^{1}$ Adam Wolf, ${ }^{2}$ Lorelí Carranza, ${ }^{2}$ and Danielle Wattsirst ${ }^{2}$

${ }^{1}$ National Renewable Energy Laboratory

${ }^{2}$ Arable Labs, Inc.

\section{Suggested Citation}

Habte, Aron, Afshin Andreas, Manajit Sengupta, Ranganath Narasappa, Andy Hoke, Peter Gotseff, Ramanathan Thiagarajan, Adam Wolf, Lorelí Carranza, and Danielle Wattsirst. 2019. Low-Cost Multiparamenter Device for Solar Resource Applications. Golden, CO: National Renewable Energy Laboratory. NREL/TP-5D00-73104.

https://www.nrel.gov/docs/fy19osti/73104.pdf.

NREL is a national laboratory of the U.S. Department of Energy Office of Energy Efficiency \& Renewable Energy Operated by the Alliance for Sustainable Energy, LLC

This report is available at no cost from the National Renewable Energy Laboratory (NREL) at www.nrel.gov/publications.

Contract No. DE-AC36-08GO28308
Technical Report NREL/TP-5D00-73104 March 2019

National Renewable Energy Laboratory 15013 Denver West Parkway Golden, CO 80401 303-275-3000 • www.nrel.gov 


\section{NOTICE}

This work was authored in part by the National Renewable Energy Laboratory, operated by Alliance for Sustainable Energy, LLC, for the U.S. Department of Energy (DOE) under Contract No. DE-AC36-08GO28308. Funding provided by U.S. Department of Energy Office of Energy Efficiency and Renewable Energy Solar Energy Technologies Office. The views expressed herein do not necessarily represent the views of the DOE or the U.S. Government.

This report is available at no cost from the National Renewable Energy Laboratory (NREL) at www.nrel.gov/publications.

U.S. Department of Energy (DOE) reports produced after 1991 and a growing number of pre-1991 documents are available free via www.OSTI.gov.

Cover Photos by Dennis Schroeder: (clockwise, left to right) NREL 51934, NREL 45897, NREL 42160, NREL 45891, NREL 48097, NREL 46526.

NREL prints on paper that contains recycled content. 


\section{Acknowledgments}

We are grateful to the U.S. Department of Energy, Office of Energy Efficiency and Renewable Energy, Technology-to-Market Program for supporting this research. We acknowledge the Solar Radiation Research Laboratory team for maintaining the world's largest array of Web-accessible, continuously operating, radiometric measurements at the National Renewable Energy

Laboratory. 


\section{List of Acronyms}

GHI

NIST

NREL

PV

SRRL

SZA

USB

UUT
Global horizontal irradiance

National Institute of Standards and Technology

National Renewable Energy Laboratory

Photovoltaic

Solar Radiation Research Laboratory

Solar zenith angle

Universal Serial Bus

Unit under test 


\section{Table of Contents}

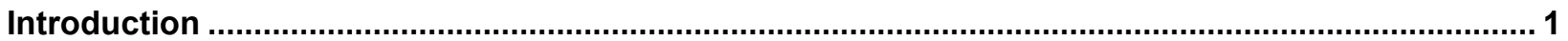

1 Objective

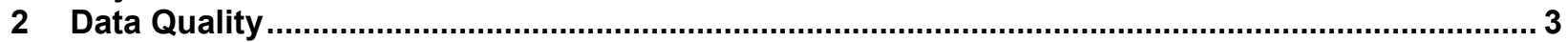

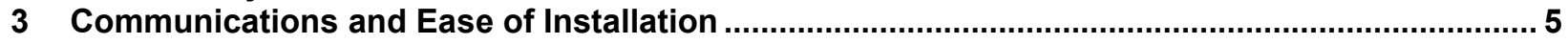

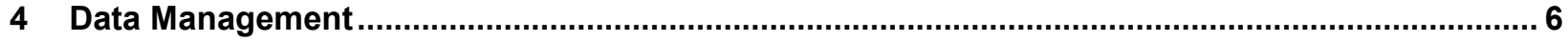

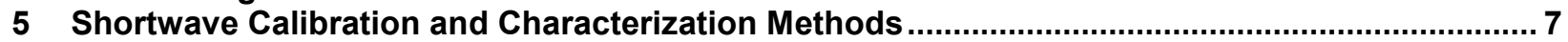

5.1 Shortwave Calibration........................................................................................ 7

5.2 Diffuse Characterization ......................................................................... 7

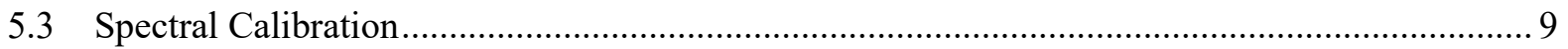

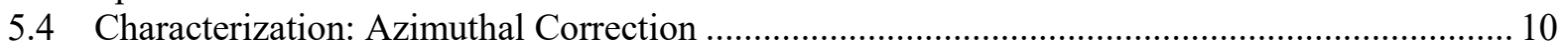

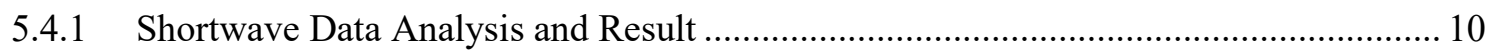

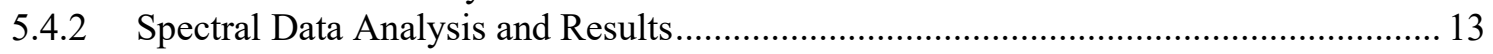

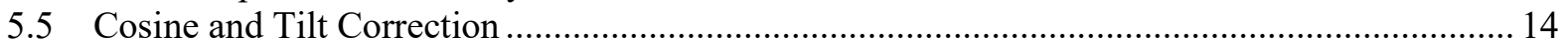

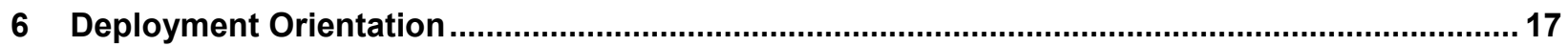

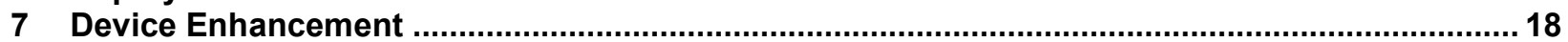

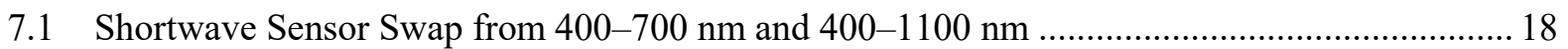

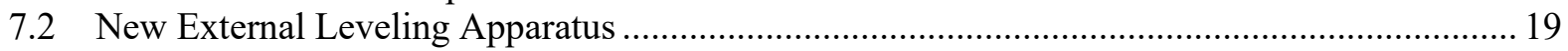

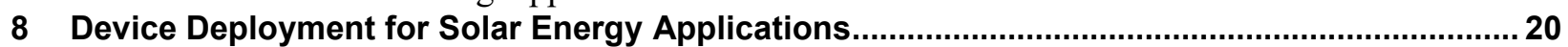

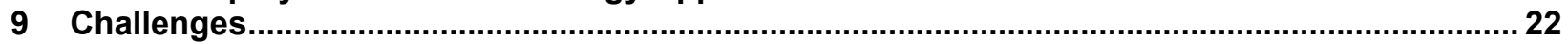

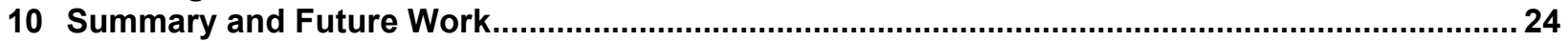

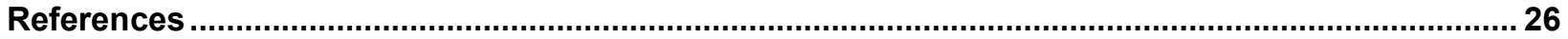




\section{List of Figures}

Figure 1. Snow on top of Arable's Mark device (left) and SRRL radiometers (right) on one winter morning after snowfall. Photos by Aron Habte, NREL ......................................................... 3

Figure 2. Voltage reading change for 2 days from Arable's Mark device $\mathrm{S} / \mathrm{N}$ : A001478 compared to the irradiance measurement from Kipp \& Zonen model CMP22 pyranometer ............................. 4

Figure 3. Arable's Mark low-cost multiparameter system at the NREL SRRL. Photos by Aron Habte,

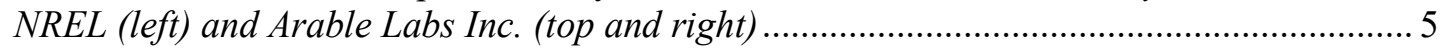

Figure 4. Arable's Mark indoor diffuser transmission........................................................... 8

Figure 5. Dark blue: diffuser mounted horizontal in the typical orientation (diffuser pointed south); red and green: diffuser mounted horizontal but rotated -90 or $90^{\circ}$; light blue: diffuser mounted near sunrise or sunset (simulated), rotated to the side and then tilted to the north to imitate the solar ray incident at an oblique angle to the surface of the diffuser.................................. 9

Figure 6. Mark detector spectral response as provided by Arable. Image modified from Arable Labs Inc. 9

Figure 7. Measured irradiance from the reference data set and the UUT as well as directional bias. The data from September 2, 2017, are used to calibrate the UUT using Eq. 1 and characterized using Eq. 2.

Figure 8. GHI measured with Arable's Mark (labeled "SRRL AMTT: Global (NREL-cor)") or with usual thermopile or photodiode radiometers during a clear day at the SRRL .............................. 11

Figure 9. Irradiance comparison of photodiode sensors relative to the reference data (1-min data set from August 18, 2017, to November 28, 2017. Each green box represents a $10^{\circ}$ bin of the interquartile range of the data in each bin. The circle in each box is a mean, and the black line indicates the median value. Ninety-five percent of the data set is within the whiskers; data points outside the whiskers are plotted with a symbol (dots).

Figure 10. One-minute GHI comparison between Arable's Mark and a reference CMP22 thermopile pyranometer

Figure 11. Spectral comparison between the WISER and Arable's Mark for January 4, 2018, at 12:00 p.m. Local Sidereal Time (left); broadband direct (green), global (red), and diffuse (blue) irradiance measured with thermopile radiometers for that clear day (right)

Figure 12. Spectral comparison between the WISER and Arable's Mark for January 20, 2018, at 12:00 p.m. Local Sidereal Time (left); broadband direct (green), global (red), and diffuse (blue)

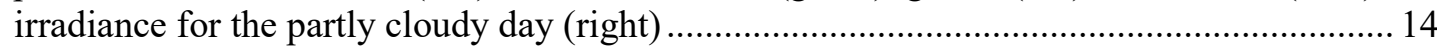

Figure 13. Extrapolating signal from Arable's Mark shortwave sensor to zero SZA............................. 15 Figure 14. Directional and tilt dependencies. The blue line shows the morning and afternoon and the red dashed line shows the power function fit.

Figure 15. One-minute GHI comparison between Arable's Mark and a reference CMP22 thermopile pyranometer after implementing the cosine and tilt correction methodology.....

Figure 16. Arable's Mark deployed in various orientations at the SRRL: 1-axis (left), fixed tilt (middle), and 2-axis (right). Photos by Aron Habte, NREL

Figure 17. NREL upgraded the shortwave silicon photodiode sensor in the device from a spectral irradiance wavelength of 400-700 nm to 400-1100 nm. Photos by Peter Gotseff, NREL .... 18

Figure 18. Design and image of Arable's new Mark leveling apparatus. Photo by Afshin Andreas (Left; design) and Aron Habte (right), NREL

Figure 19. Before applying Eq. 3 (left) and after applying the correction equation (right)..... 20

Figure 20. Comparison of measurement: red is AC power from the PV module, blue is the corrected Arable irradiance, orange is the uncorrected Arable irradiance, and green is the PV reference cell. The top panel shows a magnified view for a clear day, and the bottom panel shows a few days under all sky conditions.

Figure 21. Discoloring of Arable's Mark top surface (left) compared to a newly deployed Arable Mark (right), Photo by Aron Habte, NREL 


\section{List of Tables}

Table 1. Computed Calibration Factors for the Mark spectral sensors on September 22, 2018 ............... 10 


\section{Introduction}

The Solar Radiation Research Laboratory (SRRL) at the National Renewable Energy Laboratory (NREL), in collaboration with Arable Labs Inc., deployed, calibrated, and characterized Arable Lab's Mark low-cost, multiparameter sensor system. Arable Labs was selected by the U.S. Department of Energy for a Small Business Voucher to work with NREL to tap into the knowledge and expertise Arable needs to tailor and evaluate the Mark device for broader solar energy applications. The device is a complete and independent unit. It is a self-powered, off-theshelf solution for remote solar irradiance and meteorological sensing and data collection. Currently, the device is deployed mainly for agricultural use to assist in operational decisions, such as crop yield calculations and supply chain decisions, but it is designed to have significant potential applications, such as measuring and monitoring solar radiation elements needed by electric utilities and solar power system integrators to adequately characterize the spatial and temporal variations of solar energy generation [2].

Traceable and high-quality solar resource data are essential for many solar energy project phases, from performing feasibility studies, to due diligence during engineering design, to financing, and day-to-day plant operations [1]. Advances in grid integration and photovoltaic (PV) reliability require the development of innovative devices to provide relevant, accurate, and timely information at low cost. Ground-based monitoring stations provide traceable and high-quality solar resource data; however, they are expensive to commission and maintain. Arable's Mark device contains a unique suite of sensors to measure the downwelling and upwelling shortwave solar resource and longwave radiation, humidity, air temperature, ground temperature, precipitation, GPS, compass and tilt, and six downward- and six upward-facing narrowband spectrometer channels [2]. Thus, calibrating and characterizing Arable's Mark device at the SRRL is desirable because the SRRL has excellent solar access because of its unrestricted view of the horizon from sunrise to sunset throughout the year.

The SRRL is the home of the world's largest collection of radiometers in continuous operation and high-quality long-term solar radiation data sets. Calibration of devices against the SRRL instruments provides an understanding of the quality of a new instrument compared to peers. The characterization process provides an understanding of the variability compared to high-quality instruments and essentially provides methods to correct the sources of the variabilities. 


\section{Objective}

As stated in the introduction, the current use of Arable's Mark device is for agricultural purposes. NREL and Arable worked together to characterize and improve the company's instrumentation and measurement systems available for measuring and monitoring solar radiation elements needed by electric utilities and solar power system integrators. For this purpose, Arable provided Mark devices for evaluation. The tasks included installation, operating, calibration, and maintenance for a low-cost sensor for solar resource assessment at the SRRL for demonstrating ease of installation, system value to stakeholders, and validation of measurement accuracy by comparison with well-characterized instruments in the SRRL Baseline Measurement System. Specific tasks include:

1. Perform an outdoor calibration and characterization.

2. Identify measurement biases of the system and develop correction methodologies.

3. Publish and implement the calibration and characterization methodology. 


\section{Data Quality}

SRRL staff carry out proper operation and maintenance 5 days per week except on weekends and holidays for all devices, including Arable's Mark [3]. As stated in [1], [3], proper operation and maintenance assists in obtaining accurate solar resource measurements. For example, regular cleaning prevents attenuation of the signal through time because of soiling and removing snow from the device optics reduces bad data (Figure 1).
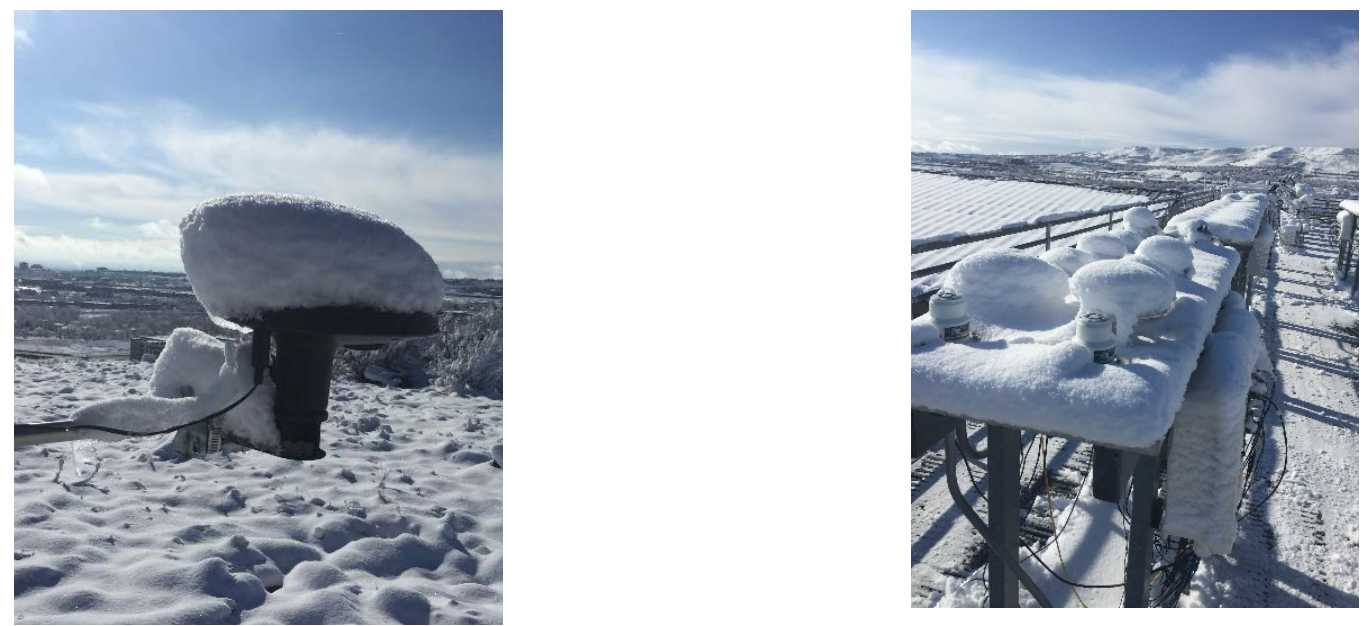

Figure 1. Snow on top of Arable's Mark device (left) and SRRL radiometers (right) on one winter morning after snowfall. Photos by Aron Habte, NREL

Multiple issues were discovered that impacted the data quality during the deployment period of Arable's Mark devices. These problems were related to equipment and operational errors, including communications issues, sudden changes in voltage readings, firmware issues, and unknown issues. Most of these problems were discovered and resolved appropriately. For instance, in October 2018, an increase in the voltage reading ( $\approx 4 \%$ change) from Arable's Mark device was observed. It is unknown what triggered the issue; however, this necessitated a new calibration and characterization (Figure 2). 

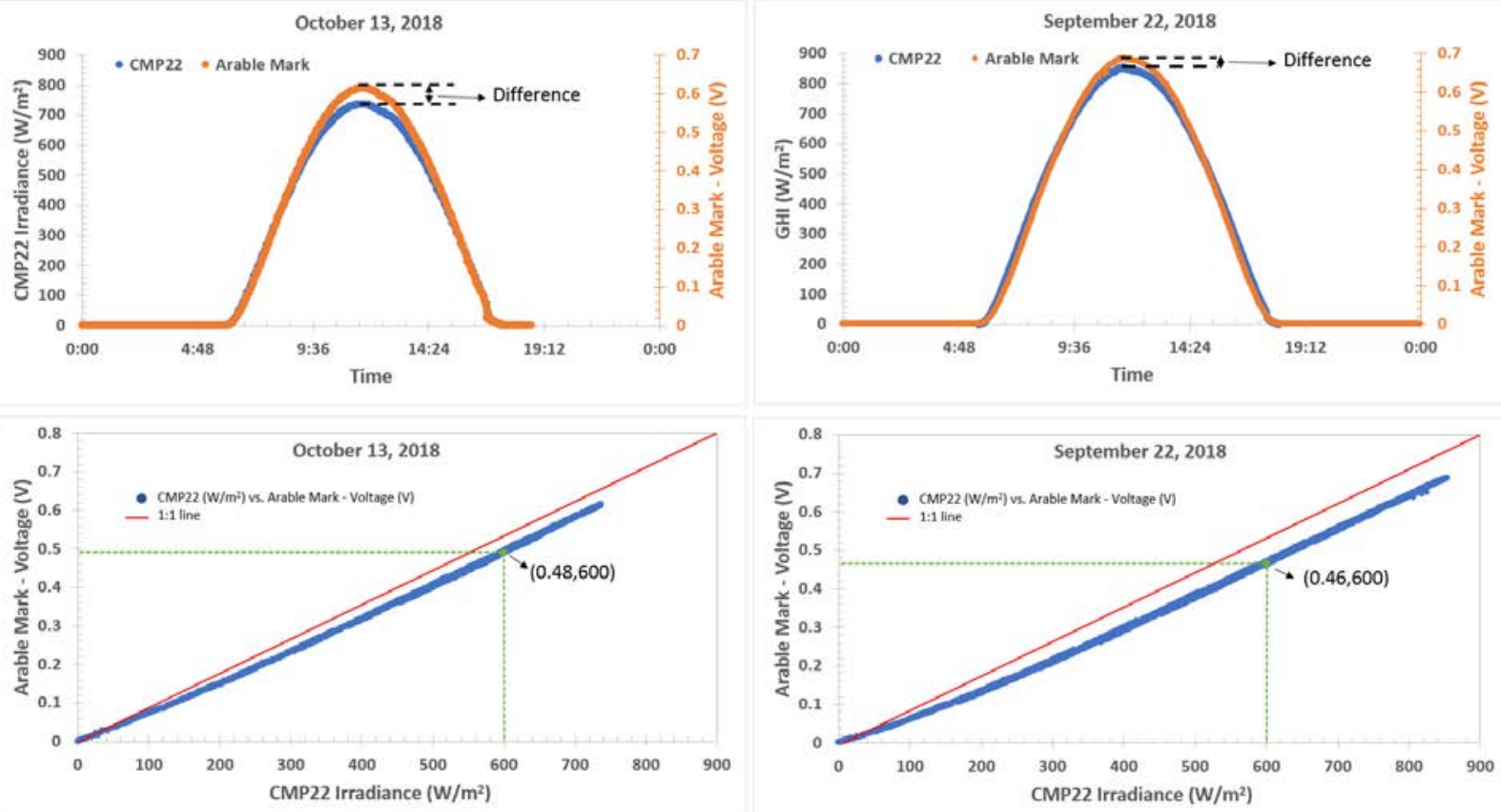

Figure 2. Voltage reading change for 2 days from Arable's Mark device S/N: A001478 compared to the irradiance measurement from Kipp \& Zonen model CMP22 pyranometer 


\section{Communications and Ease of Installation}

Arable's Mark uses a cellular communications system to facilitate the real-time availability of data, and it includes a Global Positioning System for synchronizing time and identifying its location. Additional features include an embedded solar panel, innovative mounting options, highly simplified connectors, and minimal user configuration for easy installation in remote areas.

In most cases, Arable's Mark data are transmitted and collected through cloud services via a Cloud Application Programming Interface. Additionally, at SRRL, a direct communications option was used by deploying a J-link cable (see Section 4 for more information).

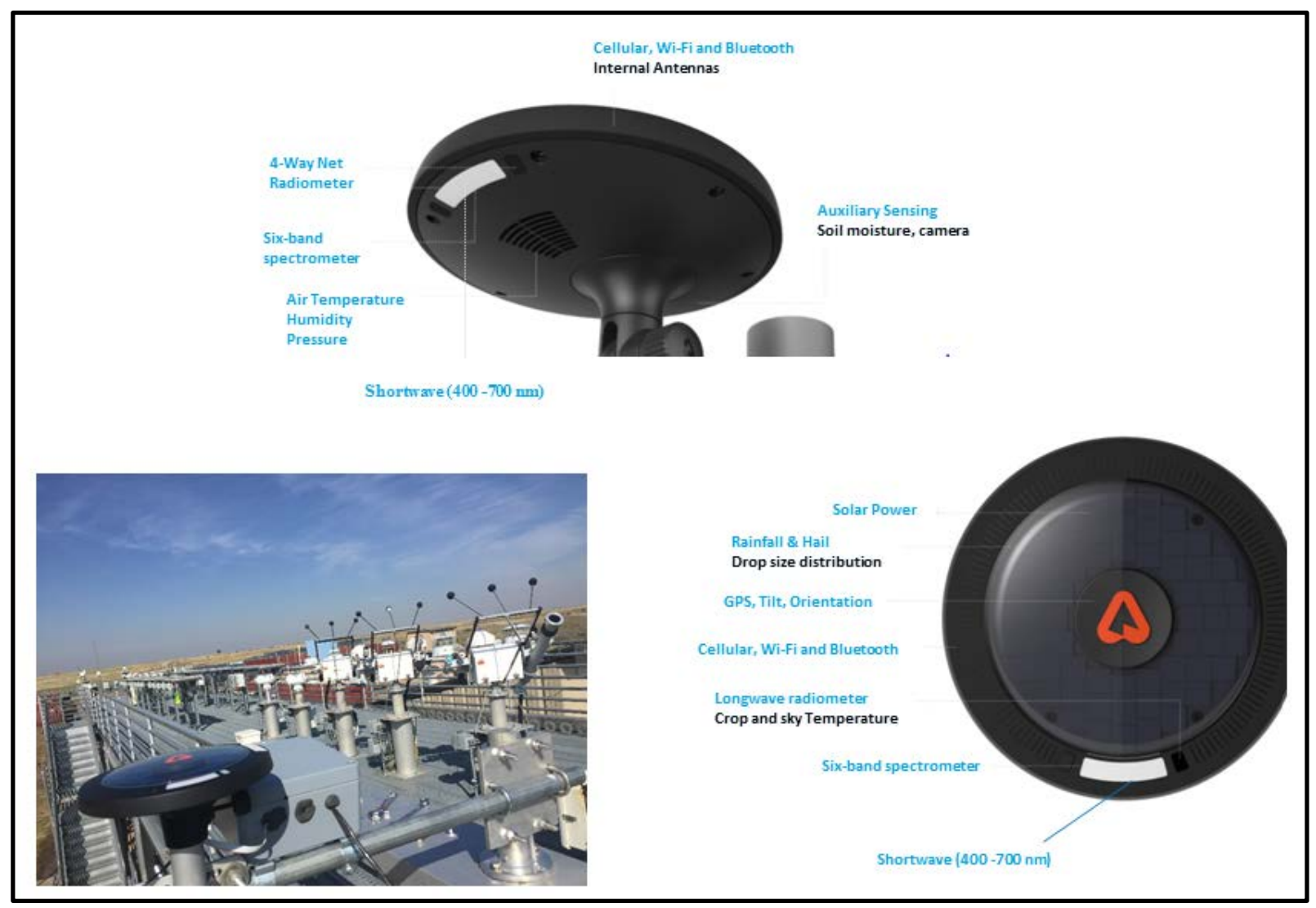

Figure 3. Arable's Mark low-cost multiparameter system at the NREL SRRL. Photos by Aron Habte, NREL (left) and Arable Labs Inc. (top and right) 


\section{Data Management}

Arable designed the Mark such that data are uploaded to the Arable cloud via cell modem. The end user is then able to access the data from the cloud; however, this does not occur in real time, and the Mark might post data to the cloud only once per hour. In some applications, the user might want direct access to the sensor output values for real-time applications. Arable did not have this feature in mind when the Mark was designed; however, NREL had a strong desire to implement such a feature. A makeshift feature that Arable provided to NREL was to use a J-link device (manufactured by SEGGER), which is primarily used at the factory to write firmware to the device and collect calibration coefficients for radiometers. The J-link is meant for temporary indoor use, but we were able to use it long term outdoors. This required drilling a hole in the Mark's mount for the J-link cable to exit the Mark. The J-link then needed to be placed in a weatherproof box, which was difficult to mount because of the short length of the J-link cable. The J-link device that Arable provided us had a Universal Serial Bus (USB) output for connection to a computer (though a more expensive J-link is available with Ethernet connection). The computer was placed indoors with a special long USB extension cable to reach the Mark, which was mounted outdoors on the SRRL instrument platform. To communicate with the JLink, the SEGGER software package needed to be installed on the computer. This software provides a driver for the USB interface (Ethernet versions would not require this). In addition, the J-Link Commander software must always be running in the background. Once the J-Link Commander is running, the end user can write their own software to read data over a Transmission Control Protocol/Internet Protocol port on the computer using a telnet-type client. NREL wrote software in Perl to automatically read the data from the Mark's 1-minute real-time data output stream and reformat the data for compatibility for NREL's Measurement and Instrumentation Data Center website. Through Measurement and Instrumentation Data Center post-processing, NREL also applied calibration factors to the raw data reported from the Mark. 


\section{Shortwave Calibration and Characterization Methods}

\subsection{Shortwave Calibration}

Arable's Mark shortwave sensor measures global horizontal irradiance (GHI) in the spectral range of 400-700 $\mathrm{nm}$. The shortwave sensor is initially calibrated in the factory before shipment. Nevertheless, after installation at the SRRL, the sensor was recalibrated using NREL's Broadband Outdoor Radiometer Calibration methodology. The calibration was carried out using the SRRL's reference direct normal irradiance and diffuse horizontal irradiance radiometers. As stated in [3], the reference GHI (GHIref) is obtained from the direct normal irradiance and diffuse horizontal irradiance using the component-sum procedure. The data from these reference radiometers are traceable to the System International units through the World Radiation Reference scale [1]-[3]. Both reference radiometers - a Kipp \& Zonen model CHP1 pyrheliometer and a shaded Eppley Laboratory 8-48 pyranometer-were chosen because of their low combined uncertainty [3].

Photodiode sensors such as those used in Arable's Mark shortwave sensor can be calibrated using NREL's SRRL process by following one of two options: (1) calibration as a function of solar zenith angle (SZA) or (2) calibration at a fixed SZA of $45^{\circ}$ [3]. For the specific sensor under scrutiny here, however, an optimum calibration SZA of $37.5^{\circ}$ was selected, and the calibration factor was derived using:

$$
R=\frac{V}{G H I_{\text {ref }}}
$$

where $\mathrm{R}$ is the instrument's responsivity, in $\mu \mathrm{V} /\left(\mathrm{W} \mathrm{m}^{2}\right)$, and $\mathrm{V}$ is the instrument's sensor output voltage $(\mu \mathrm{V})$.

The possible reason for the optimum calibration SZA of $37.5^{\circ}$ is because the directional and tilt issues of the device become minimal when the sun is high, e.g., $37.5^{\circ}$.

In addition to outdoor calibration, NREL obtained preliminary data from the Mark that were taken indoors for comparison with outdoor calibration results. The Mark was set up in NREL's Optical Metrology Laboratory oriented in a vertical position at $50 \mathrm{~cm}$ from a National Institute of Standards and Technology (NIST) Standard of Spectral Irradiance. Using the data provided by NIST, the user has the spectral irradiance from 250-2400 nm at every wavelength. Therefore, the spectral channels from the Mark could also be calibrated in this manner. For the broadband irradiance, NIST does not supply data beyond $2400 \mathrm{~nm}$. Therefore, an EKO MS-80 thermopile pyranometer, which has a negligible thermal offset, was used to measure the total broadband irradiance from the NIST lamp. The EKO MS-80 was calibrated through NREL's Broadband Outdoor Radiometer Calibration process. Using the broadband irradiance reported from the MS80 , the shortwave sensor could be calibrated.

\subsection{Diffuse Characterization}

Prior to conducting any spectral calibration of the detectors in the Mark, the spectral transmission characteristics of the diffuser needed to be understood. The primary concern was to 
confirm that the diffuser was transmitting all wavelength regions necessary for the spectral and shortwave detectors. The other concern was to determine if the relative spectral transmission curve changes as a function of solar incidence angle.

For the transmission testing, the top cover of the Mark was removed such that light could be transmitted through the diffuser to an NREL spectroradiometer on the receiving end. For the absolute spectral transmission at normal incident, a stable DC light source (indoors) was first measured with an ASD FieldSpec Pro spectroradiometer. Then the diffuser was inserted into the light path at normal incidence, and the ratio of light with the diffuser and light without the diffuser was computed to produce the transmission curve shown in Figure 4.

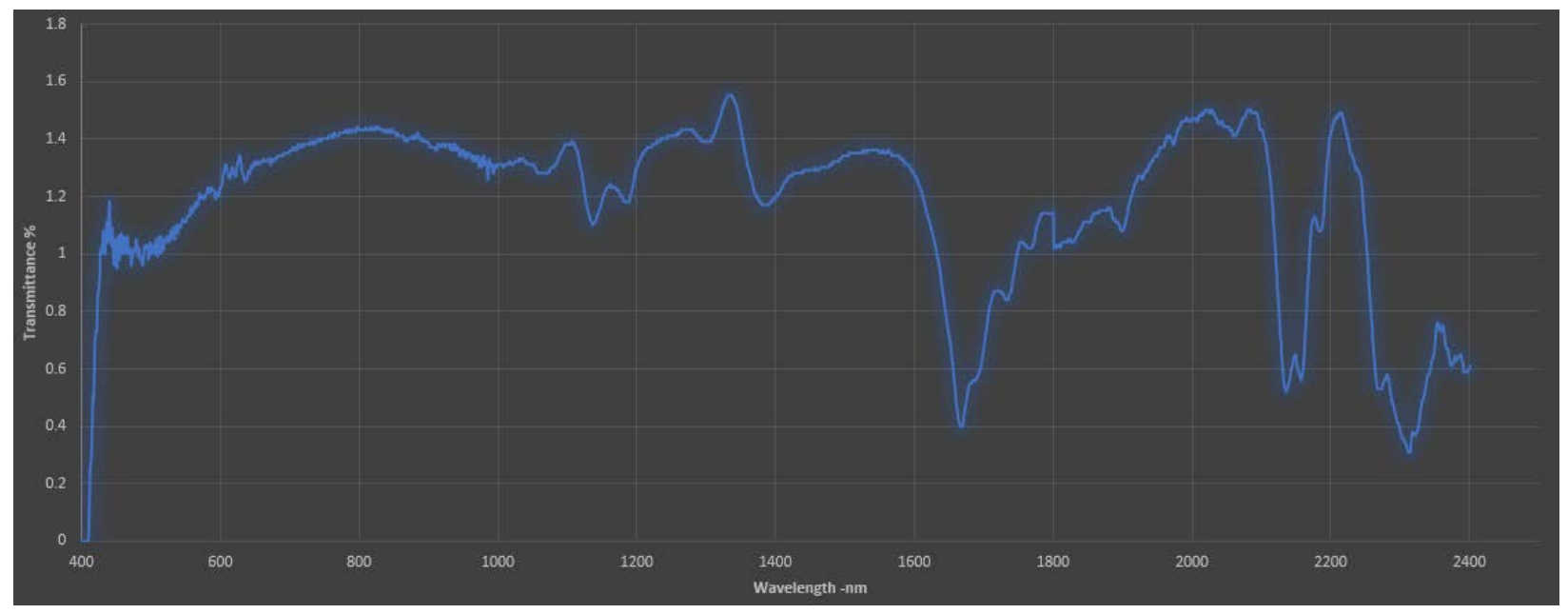

Figure 4. Arable's Mark indoor diffuser transmission

Further, outdoor spectral transmission as a function of incidence angle was tested by comparing the spectral transmission curve at various angles with respect to the spectral transmission in the normal orientation. For perfect transmission, the ratio would be flat in the region of the shortwave and spectral detectors of the Mark. This testing was done outdoors to avoid any geometry issues associated with indoor setups. A bracket was built to hold the ASD spectroradiometer input fiber probe to the Mark's diffuser so that it would always be in the same place when the diffuser is moved to different angles. To measure the normal incidence spectral irradiance, the diffuser was placed on the front surface of an outdoor solar tracker held normal to the sun. Then the tracker and diffuser were moved to four other orientations, and the spectral irradiance was measured at those locations. Finally, the ratio was computed for each orientation with respect to the normal orientation, as shown in Figure 5. 


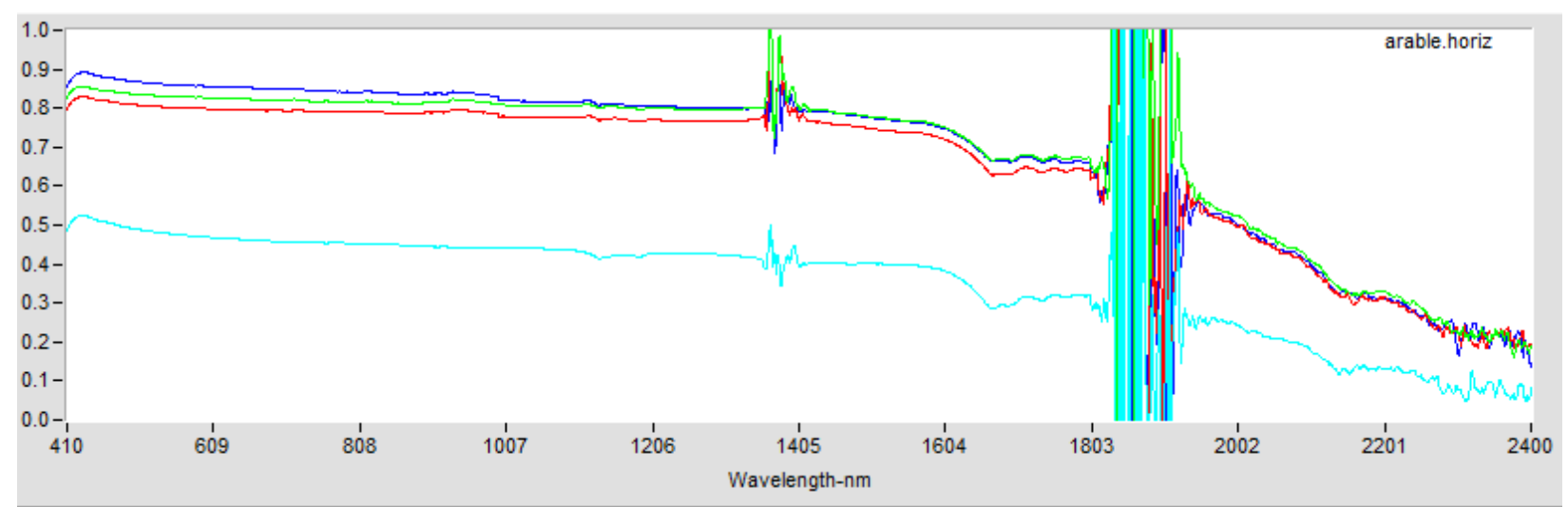

Figure 5. Dark blue: diffuser mounted horizontal in the typical orientation (diffuser pointed south); red and green: diffuser mounted horizontal but rotated -90 or $90^{\circ}$; light blue: diffuser mounted near sunrise or sunset (simulated), rotated to the side and then tilted to the north to imitate the solar ray incident at an oblique angle to the surface of the diffuser

As shown in Figure 5, the diffuser has near flat transmission with slight decline up to $1600 \mathrm{~nm}$.

\subsection{Spectral Calibration}

Narrowband radiometers are typically calibrated under conditions where radiometers are normally used to avoid spectral mismatch errors. Therefore, the Mark spectral detectors were calibrated outdoors against an EKO Instruments WISER spectroradiometer system that is deployed in continuous operation at the SRRL. The WISER is traceable to NIST and calibrated in NREL's Optical Metrology Laboratory. The manufacture provided NREL with typical spectral response used for the detectors in the Mark, as shown in Figure 6. The shape of each detector is different, and the bandwidth is rather large for some applications.

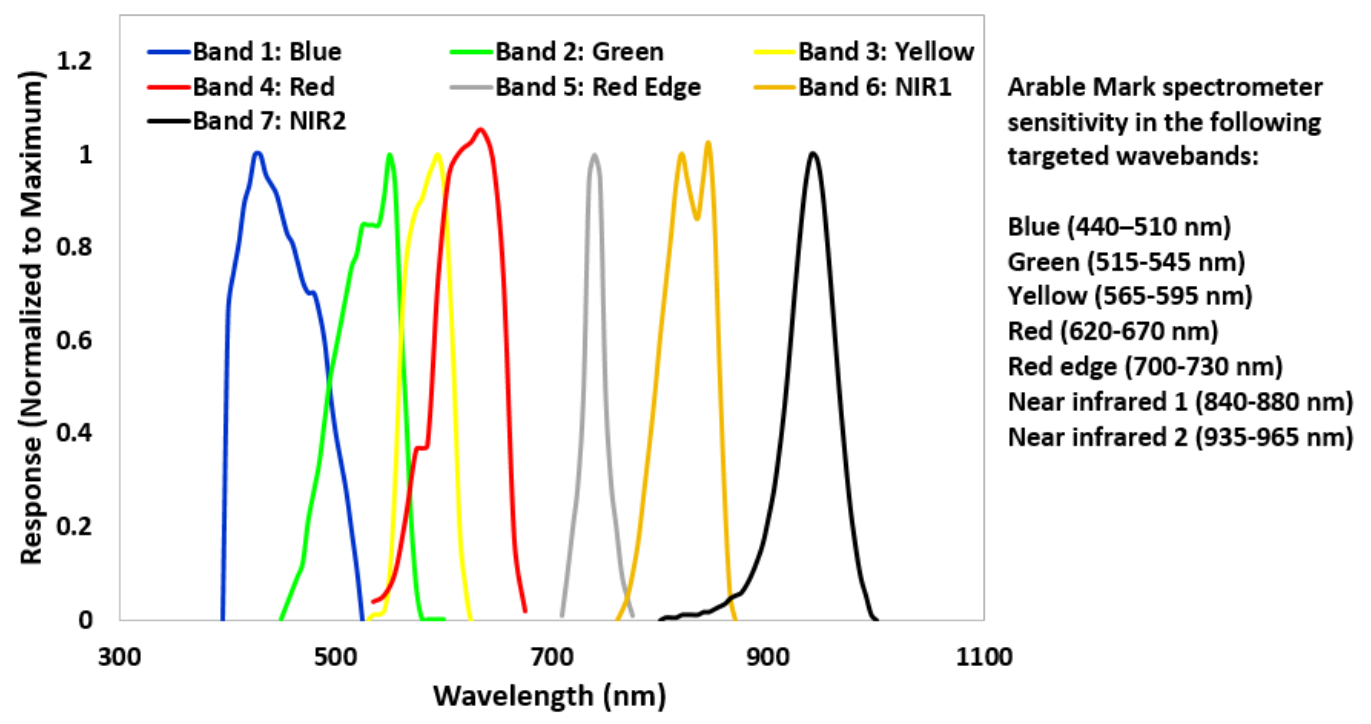

Figure 6. Mark detector spectral response as provided by Arable. Image modified from Arable Labs Inc. 
NREL decided to treat the detectors as a single point at the middle of the response curve, which is an ideal scenario if the sensors were later used in a model to compute the entire solar spectrum. A calibration factor for each detector can be computed by taking the reference irradiance from the WISER at the appropriate wavelength divided by the signal in volts from the detector being calibrated.

The calibration was conducted on a clear day, and the average calibration factors were computed for SZAs between $44-46^{\circ}$ for both morning and afternoon, as shown in Table 1.

Table 1. Computed Calibration Factors for the Mark spectral sensors on September 22, 2018

\begin{tabular}{|l|l|}
\hline Wavelength & Calibration Factor $\left(W / \mathbf{m}^{2} / \mathbf{n m} / \mathbf{V}\right)$ \\
\hline $\mathbf{4 7 5} \mathbf{~ n m}$ & 3.68 \\
\hline $\mathbf{5 3 0} \mathbf{~ n m}$ & 2.038 \\
\hline $\mathbf{5 8 0} \mathbf{~ n m}$ & 2.405 \\
\hline $\mathbf{6 4 5} \mathbf{~ n m}$ & 2.084 \\
\hline $\mathbf{7 1 5} \mathbf{~ n m}$ & 1.741 \\
\hline $\mathbf{8 6 0} \mathbf{~ n m}$ & 3.75 \\
\hline $\mathbf{9 5 0} \mathbf{~ n m}$ & 0.927 \\
\hline
\end{tabular}

\subsection{Characterization: Azimuthal Correction}

\subsubsection{Shortwave Data Analysis and Result}

During the measurement period, a directional dependency of the shortwave sensor was noticed (Figure 7). One of these directional dependencies is because of the azimuthal orientation of the detector with respect to the incoming solar radiation. To correct for this dependency, a correction was applied as a function of solar azimuth. To remove this error, a polynomial function of the solar azimuth was implemented using the bias line, as follows:

$$
E_{\text {cor }}=E_{\text {raw }}-\sum_{0}^{6} a_{i} A^{i}
$$

where $E_{c o r}$ is the corrected irradiance for the unit under test (UUT), $E_{\text {raw }}$ is the uncorrected (raw) irradiance, $A$ is the solar azimuth, and $a_{i}$ are numerical coefficients obtained by least-squares fitting:

- $a_{0}=10671.15$

- $a_{1}=-401.84$

- $a_{2}=6.06$

- $a_{3}=-4.74 \mathrm{E}-2$

- $a_{4}=2.038 \mathrm{E}-04$

- $a_{5}=-4.57 \mathrm{E}-07$

- $a_{6}=4.19 \mathrm{E}-10$. 


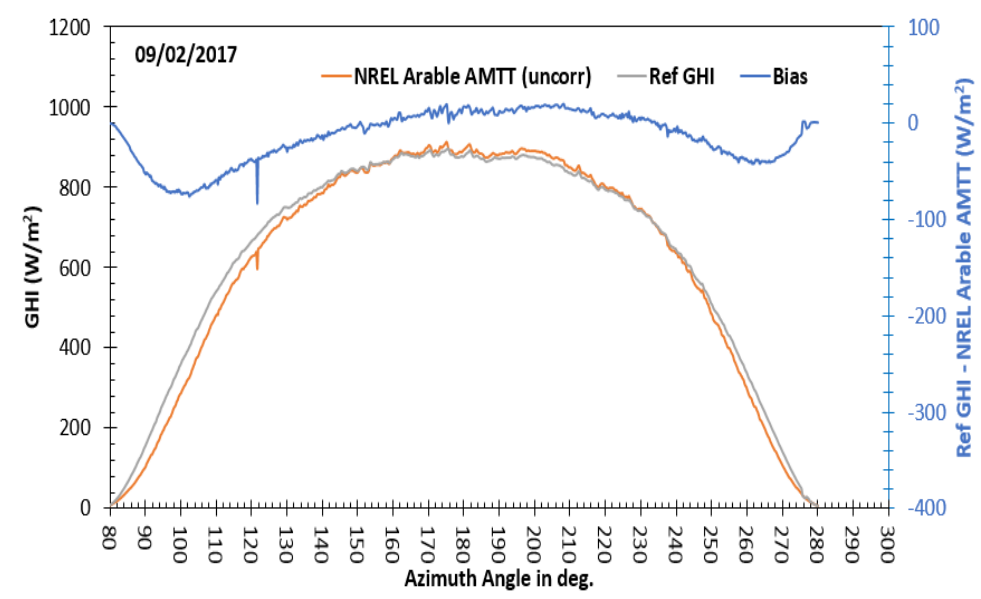

Figure 7. Measured irradiance from the reference data set and the UUT as well as directional bias. The data from September 2, 2017, are used to calibrate the UUT using Eq. 1 and characterized using Eq. 2.

Equation 2 was implemented only for SZA $<80^{\circ}$ and for any clearness index $\left(K_{t}\right.$ or $\left.K_{n}\right)$ greater than 0.3. (As usual, $K_{t}$ is defined as the ratio between GHI and its extraterrestrial counterpart; similarly, $K_{n}$ is defined as the ratio between direct normal irradiance and its extraterrestrial counterpart.) Outside of these ranges, the uncorrected UUT irradiance was used. This is because of the limitations of Eq. 2, which is imprecise at low sun elevation or undercast/overcast conditions.

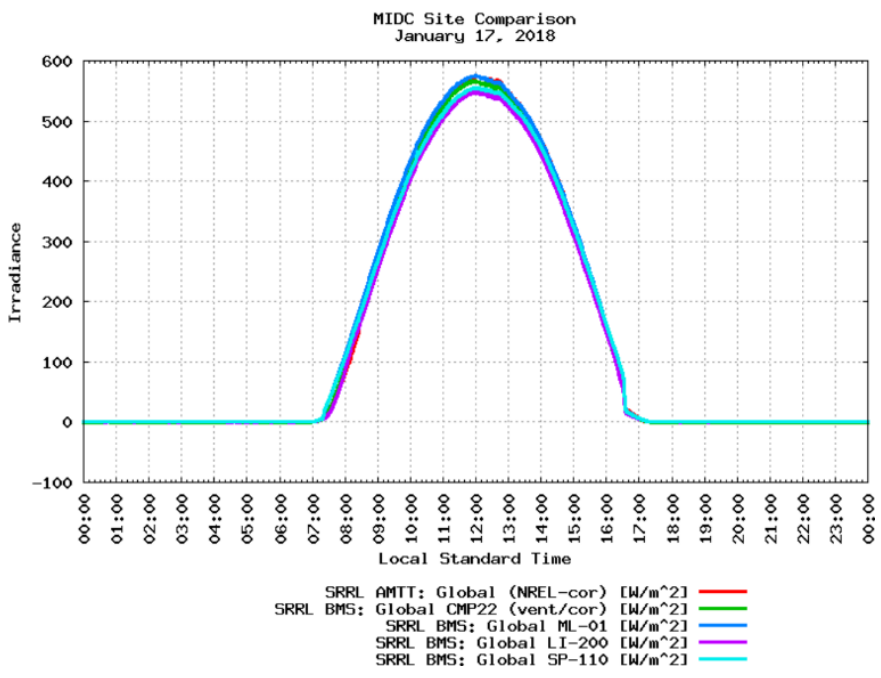

Figure 8. GHI measured with Arable's Mark (labeled "SRRL AMTT: Global (NREL-cor)") or with usual thermopile or photodiode radiometers during a clear day at the SRRL

Figure 8 shows a comparison of the corrected shortwave data using the method described here with a thermopile pyranometer (Kipp \& Zonen model CMP22) and photodiode sensors (LICOR's model LI-200, EKO's model ML-01, and Apogee's model SP-110). After correction, the UUT appears to compare well with these more conventional instruments.

To understand the difference better, 1-min clear-sky data $\left(K_{n}>0.7\right)$ from August 18 to November 28, 2017, from the photodiode pyranometers (UUT, EKO ML-01, LICOR LI-200, 
and SP-110) were compared to the reference data. As shown in Figure 9, the radiometers labeled " 1 " and "2" (x-axis) both actually represent the UUT. UUT-1 is corrected according to Eq. 1 (NREL-corrected), whereas UUT-2 refers to the original factory calibration. The NREL correction method shows $\pm 2 \%$ bias compared to $2 \%-8 \%$ when using only the factory methodology. Further, the NREL-corrected UUT shows a comparable or better result, with an average bias of less than $\pm 1 \%$ compared with the three conventional photodiode sensors. The NREL-corrected UUT also contains less directional error compared to the LICOR LI-200 unit. The interquartile range is larger for the NREL-corrected UUT, however, compared to the three sensors. This is caused by more variability and noise in the signal, apparently caused by the imperfect correction method.

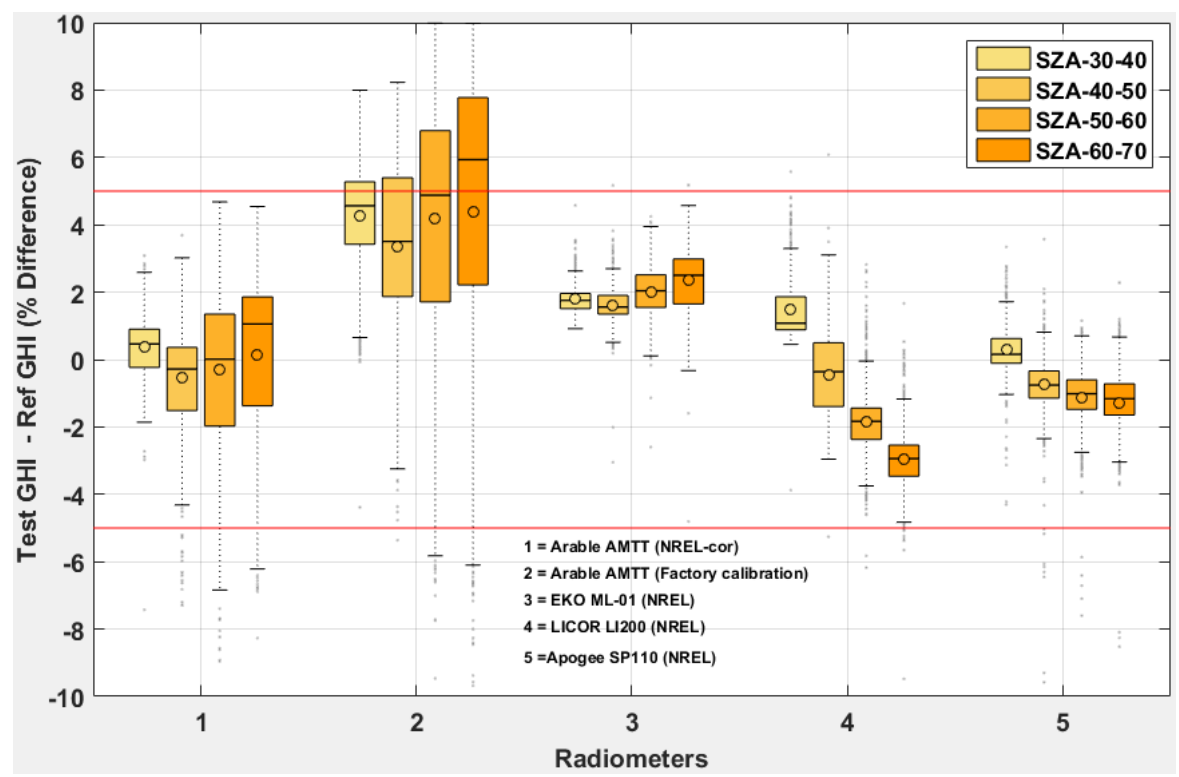

Figure 9. Irradiance comparison of photodiode sensors relative to the reference data (1-min data set from August 18, 2017, to November 28, 2017. Each green box represents a $10^{\circ}$ bin of the interquartile range of the data in each bin. The circle in each box is a mean, and the black line indicates the median value. Ninety-five percent of the data set is within the whiskers; data points outside the whiskers are plotted with a symbol (dots).

Further, a long-term analysis included an all-sky comparison at 1-minute temporal resolution during a 3-month period (August 18, 2017-November 28, 2017). The results show good agreement with an R-square value of $\sim 0.99$ compared with the reference CMP22 instrument (Figure 10). 


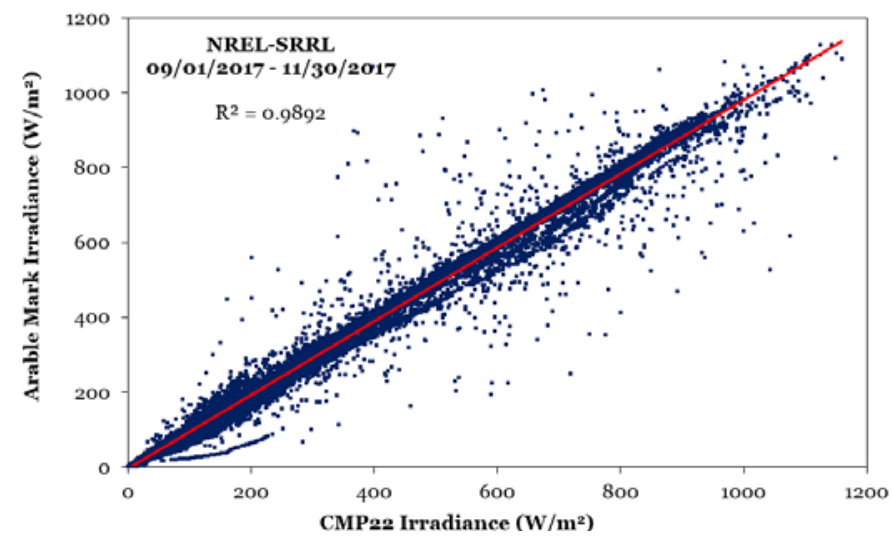

Figure 10. One-minute GHI comparison between Arable's Mark and a reference CMP22 thermopile pyranometer

\subsubsection{Spectral Data Analysis and Results}

In addition to the broadband sensor discussed above, Arable's Mark device is equipped with a six-band spectrometer (Fig. 11). These bands cover the range from 400-950 nm and were calibrated at the SRRL against an EKO WISER model MS-711 spectroradiometer, which is regularly lamp calibrated, with traceability to the NIST standard. Arable's Mark spectrometer contains azimuthal errors similar to the broadband sensor. A polynomial function similar to the one described in Section 5.4.1 is used to correct this error. After correction, the Mark and WISER instruments show good agreement under clear-sky conditions (Fig. 11).
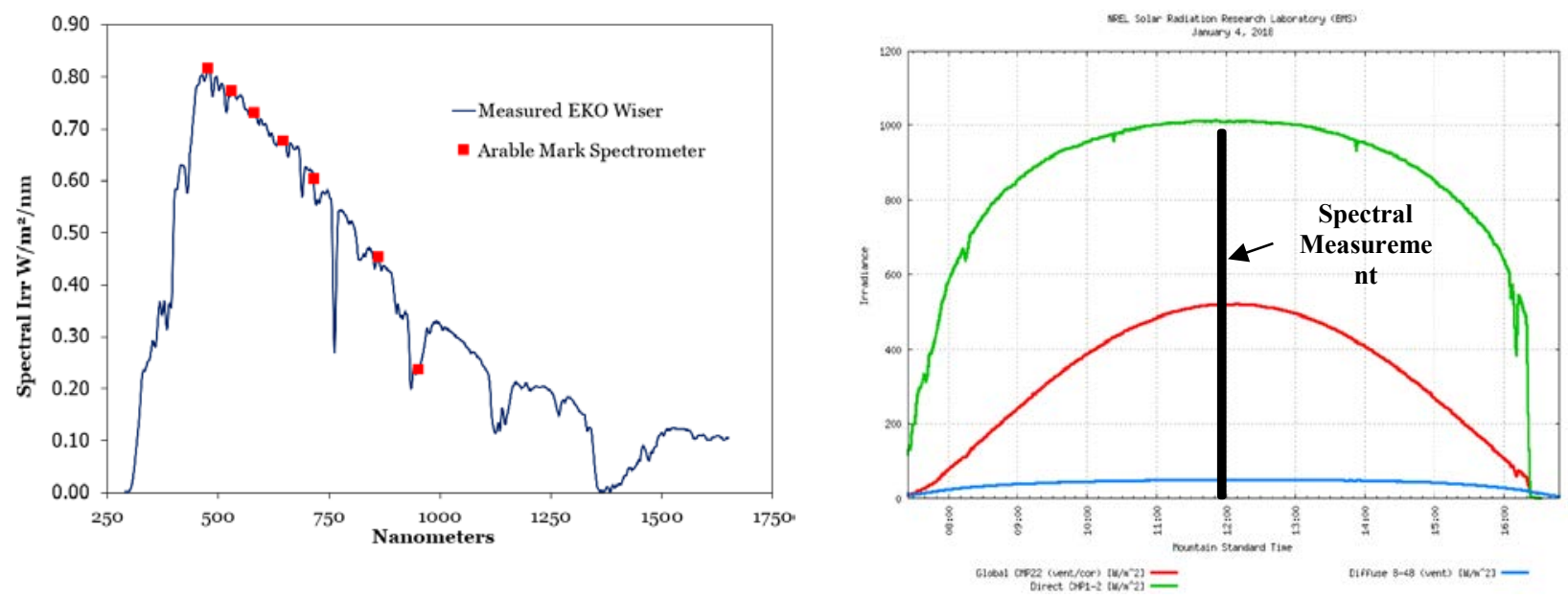

Figure 11. Spectral comparison between the WISER and Arable's Mark for January 4, 2018, at 12:00 p.m. Local Sidereal Time (left); broadband direct (green), global (red), and diffuse (blue) irradiance measured with thermopile radiometers for that clear day (right)

Under partly cloudy skies, however, the difference in spectral global irradiance sensed by the two instruments is significant. This can be partly attributed to the difference in scan rate because the WISER scans every 5 minutes, whereas the spectrometer scans each minute. Sky conditions and atmospheric transmittance can change rapidly under cloudy situations, hence triggering rapid variations in the solar spectrum (Figure 12). The Mark's rapid scan time can be an advantage in, e.g., applications involving PV spectral effects. 
Note here that the present results represent only the location, data period, and instruments specifically used for this study. It is known that different specimens of the same radiometer model might have differing characteristics and that silicon sensors are affected by spectral variations in the incident spectrum, which depend on atmospheric conditions and thus on location [4], [5]. Work is underway to better characterize these spectral effects and to reduce the measurement uncertainty of Arable's Mark with more advanced corrections.
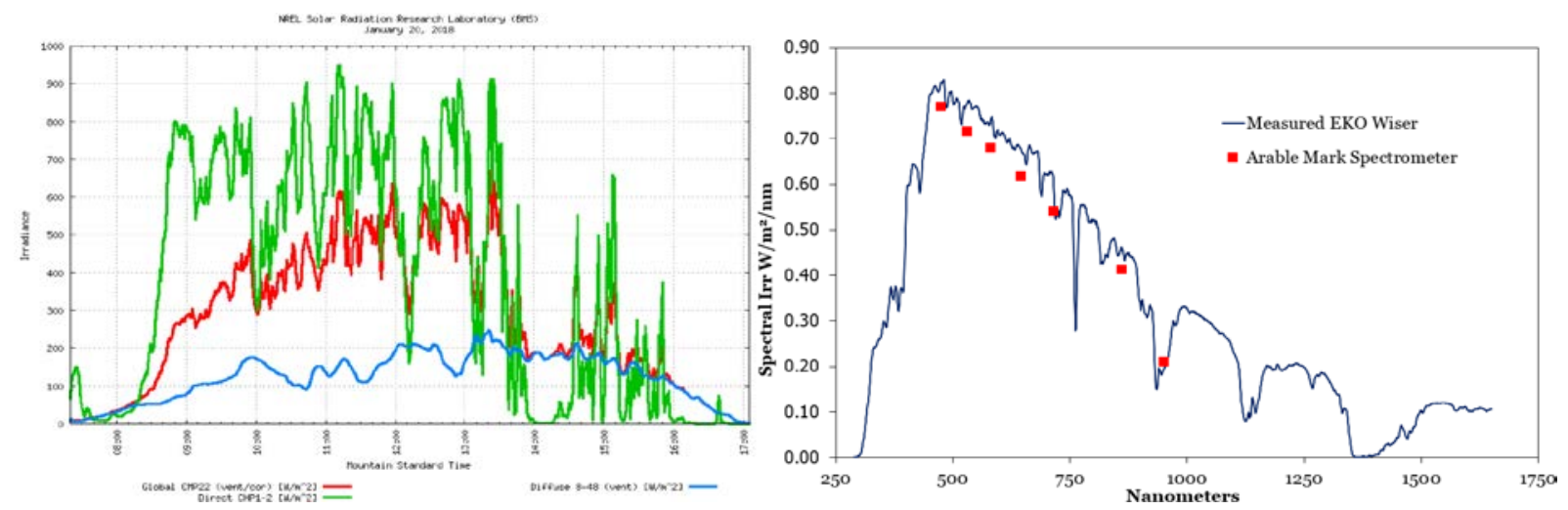

Figure 12. Spectral comparison between the WISER and Arable's Mark for January 20, 2018, at 12:00 p.m. Local Sidereal Time (left); broadband direct (green), global (red), and diffuse (blue) irradiance for the partly cloudy day (right)

\subsection{Cosine and Tilt Correction}

The azimuthal correction methodology showed limited success in providing accurate corrected irradiance throughout the year. The correction worked well when the azimuth angle of a measurement period is within the azimuth angle of the calibration and characterization period. Therefore, a new cosine and tilt correction method was developed.

To correct the cosine response error of the devices and the placement of the detector with respect to the incoming solar radiation, a correction was applied by following an approach similar to that described in [4], [5]. The correction is as a function of SZA, Eq. 3, which is then implemented in Eq. 4 and Eq. 5. This correction was obtained under a global horizontal irradiance $\left(180^{\circ}\right.$ orientation) and is applicable to any Arable Mark device. Inherent cosine errors are consistent from one device to another. As described in [4], [5], the correction obtained at NREL applies to the same model of radiometer deployed elsewhere, assuming proper installation and maintenance is maintained:

$$
\text { Rel_units }=\frac{\operatorname{Signal}(\theta)}{\operatorname{Signal}(0) * \cos (\theta)}
$$

where the signal is the voltage readings $(\mathrm{V})$, and $\theta$ is SZA in degrees. To obtain the signal reading at zero degree (denominator), the signal is plotted versus SZA and then an estimated voltage reading for zero SZA is taken from the curve $\left(\mathrm{V}_{0}=\sim 0.65 \mathrm{~V}\right)$ (Figure 13). The result of Eq. 3 is illustrated in Figure 14 and used to assist in generating the power function using curve fitting $(d)$ as described in Eq. 4 and illustrated in Figure 14 by the red dashed line.

$$
d=(a+o f f s e t) *\left(\Theta^{b}\right)+c
$$


where, a, b, c are coefficients. Two specific offset values are applied for the morning and afternoon for each device. Solar azimuth angle was used to differentiate between morning and afternoon, where solar azimuth angle less than 180 in the morning and greater in the afternoon. Further, the cosine and tilt error correction was applied only for SZAs less than $70^{\circ}$ because the cosine error correction becomes uncertain at SZAs greater than $70^{\circ}$. Therefore, for SZAs greater than $70^{\circ}$, uncorrected irradiance values were used.

Note: SZA and azimuth angles are obtained using NREL's solar positioning algorithm, which is also part of Sandia National Laboratories' PV_LIB Toolbox package.

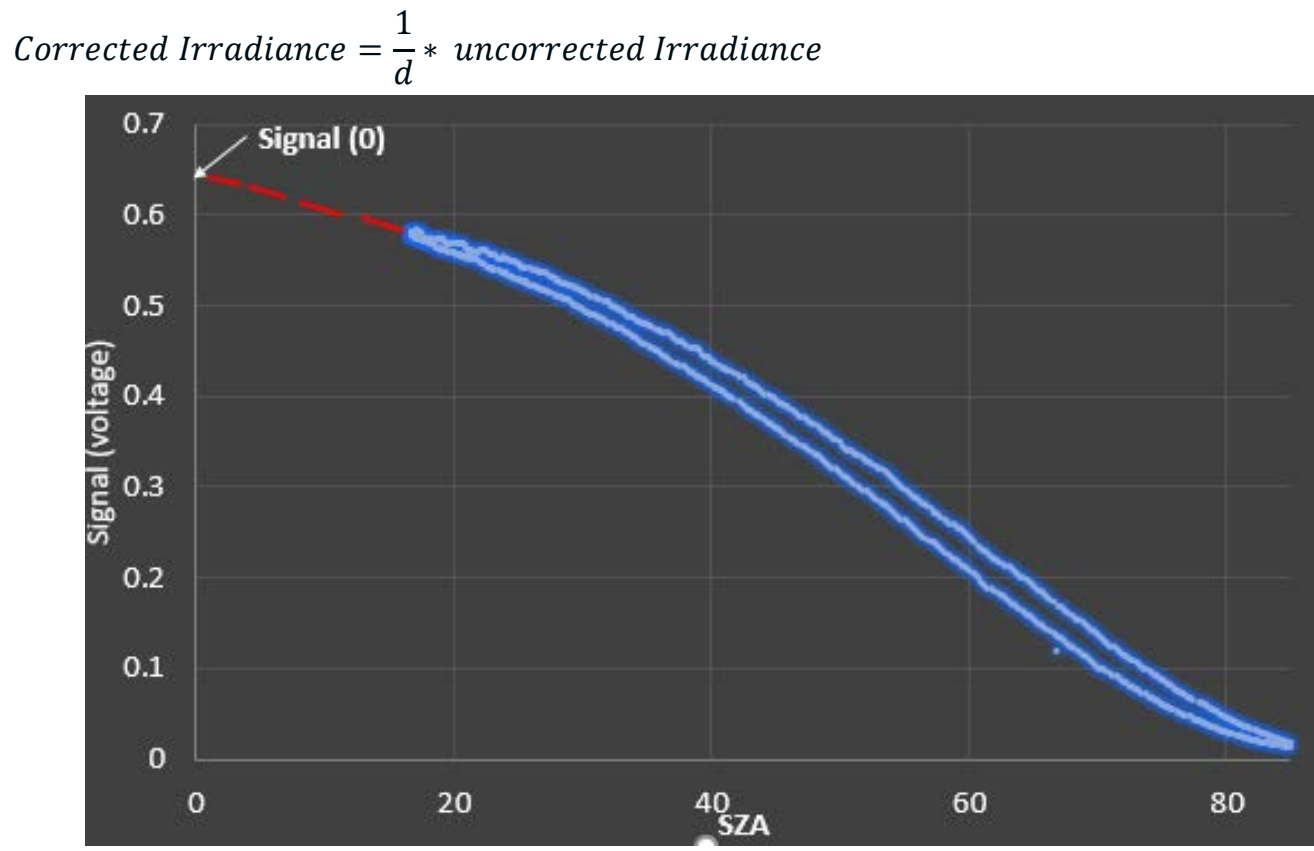

Eq. 5

Figure 13. Extrapolating signal from Arable's Mark shortwave sensor to zero SZA

If a device is perfect, Rel_units (Figure 14) is expected to be unity for all SZA; however, this is not the case for these devices because of the directional error and possible misalignment of the detector.

Figure 14 shows the device's relative directional and tilt dependencies. The two lines represent morning and afternoon readings for the calibration day. For fitting a curve in Figure 14 (note that the morning and afternoon values are obtained using Eq. 3), one can use a polynomial or some other type of fitting, but in this case we used a power function. An offset was applied to the first coefficient ("a") to compensate for/correct the morning and afternoon differences. 


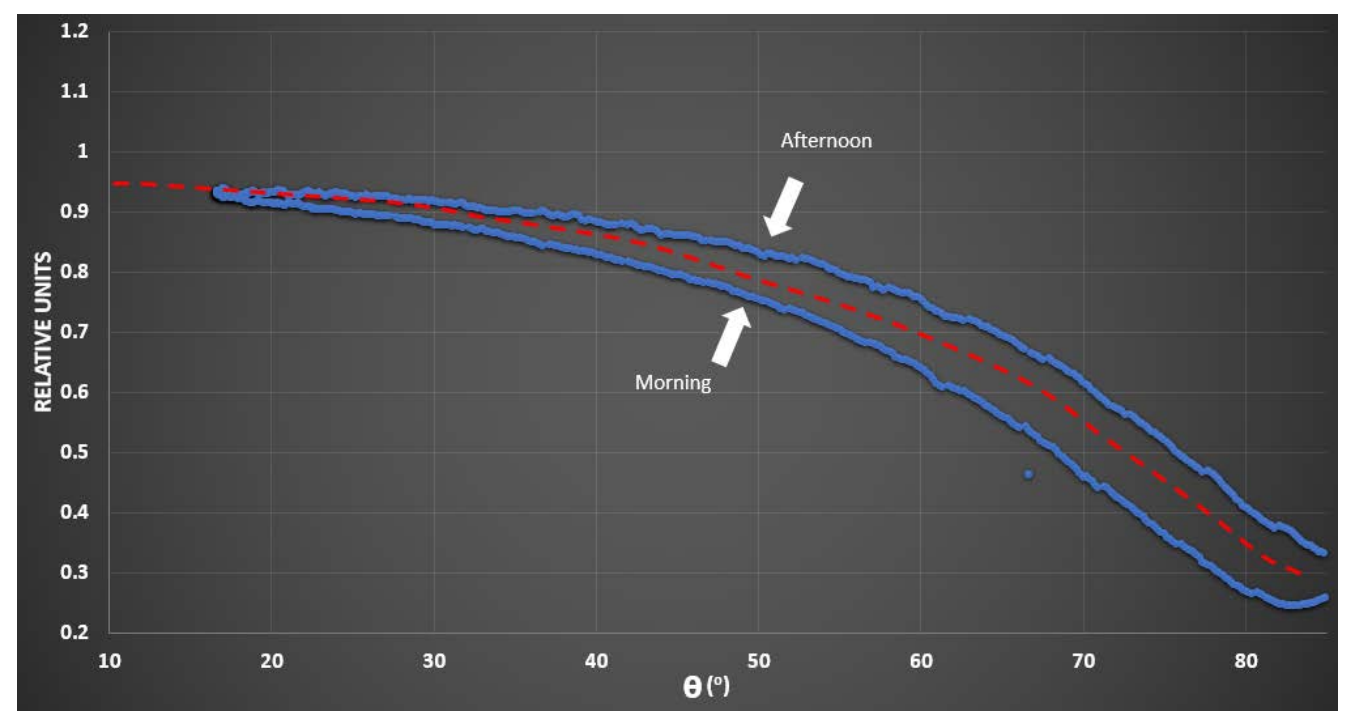

Figure 14. Directional and tilt dependencies. The blue line shows the morning and afternoon and the red dashed line shows the power function fit.

After the cosine and tilt correction, the data from Arable's Mark device demonstrated good results (Figure 15).

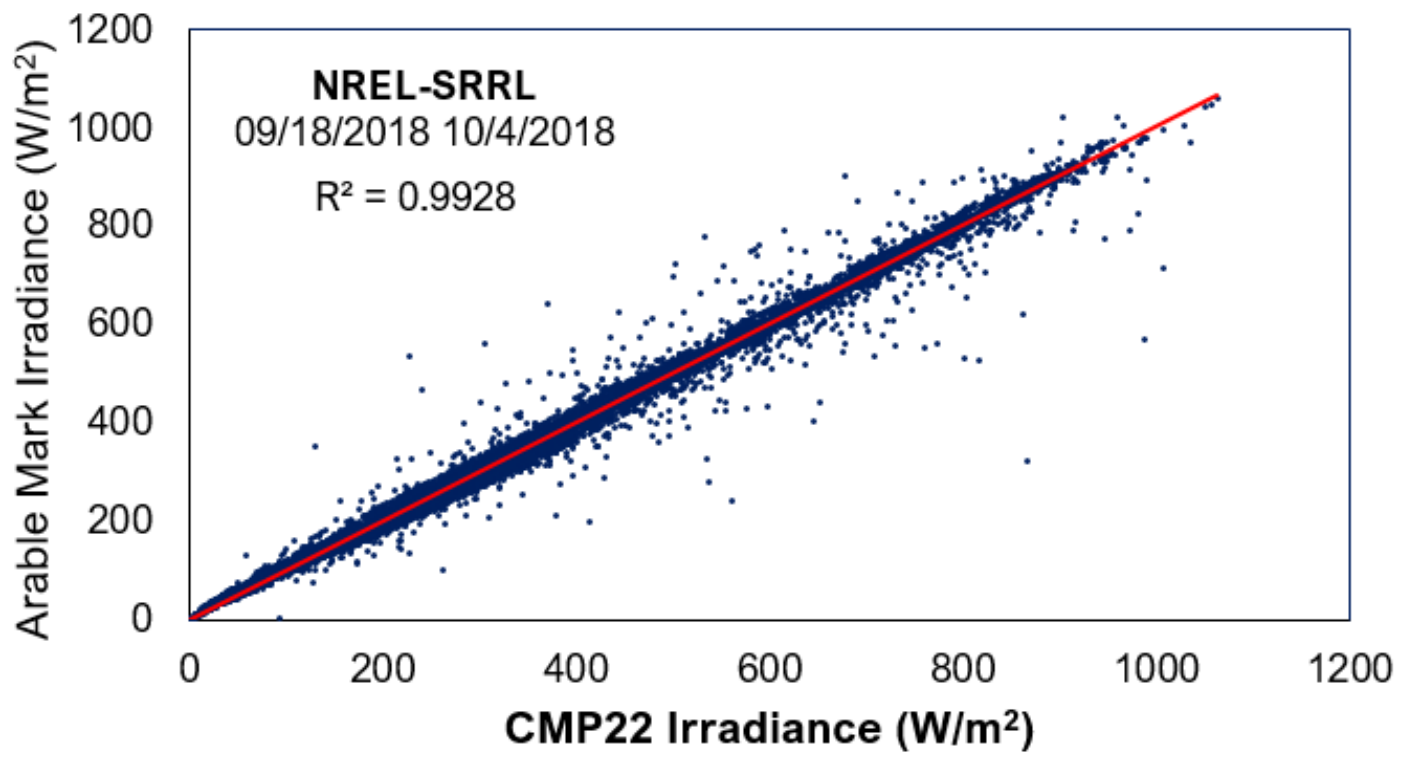

Figure 15. One-minute GHI comparison between Arable's Mark and a reference CMP22 thermopile pyranometer after implementing the cosine and tilt correction methodology 


\section{Deployment Orientation}

In addition to horizontal deployment, Arable's Mark device was installed in 1-axis and 2-axis positions. These orientations are typical solar resource measurements for PV performance applications.
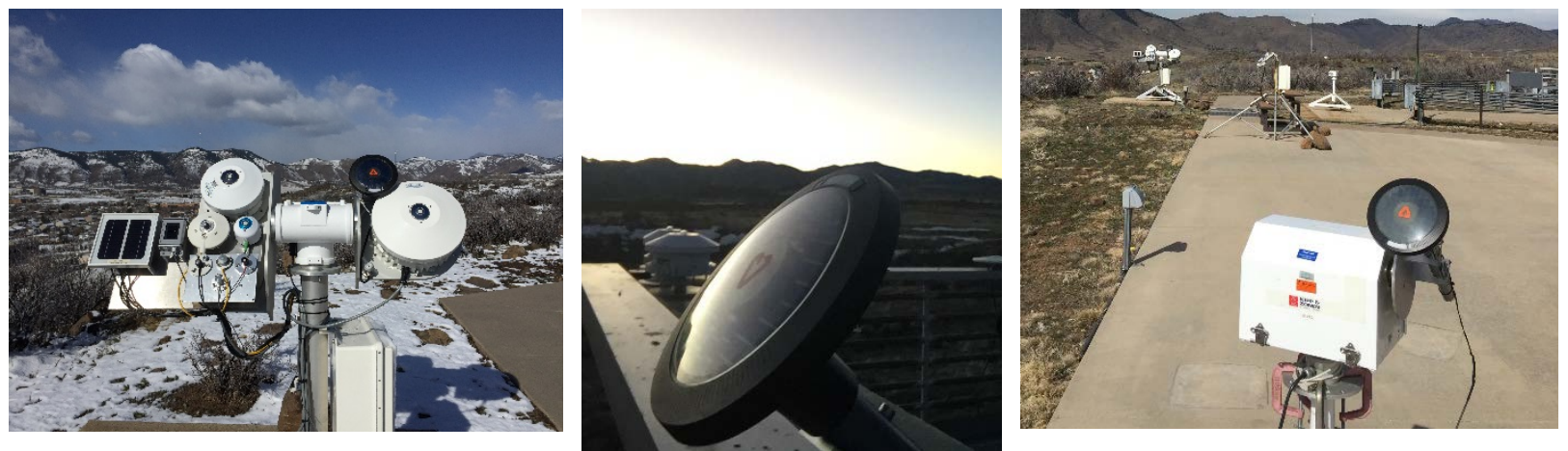

Figure 16. Arable's Mark deployed in various orientations at the SRRL: 1-axis (left), fixed tilt (middle), and 2-axis (right). Photos by Aron Habte, NREL

With proper calibration and characterization, the device is suitable for deployment under various orientations. The Arable Mark that was deployed for a Hawaiian Electric project (see Section 8) under various rooftop tilts performed well on estimating maximum available PV power at times when the PV output was curtailed. 


\section{Device Enhancement}

As stated, Arable's Mark device is designed primarily for agriculture purposes to assist in operational decisions such as crop yield calculations and supply chain decisions. With some changes in the existing device setup, however, the Mark could contribute to various solar energy project phases, including daily solar power plant operational decisions.

\subsection{Shortwave Sensor Swap from 400-700 nm and 400-1100 nm}

The shortwave sensor of Arable's Mark is equipped with silicon photodiode, which sees only the power of electromagnetic radiation in the spectral range that is used by plants for photosynthesis (400-700 nm). This is considered as photosynthetically active radiation. Most PV modules or reference PV cells, however, see in the range of 400-1100 nm. Therefore, NREL, in collaboration with Arable Labs, removed the photosynthetically active radiation sensor and installed a new silicon photodiode sensor with a range capability of 400-1100 nm. This way, the device is expected to be more applicable for solar energy applications.

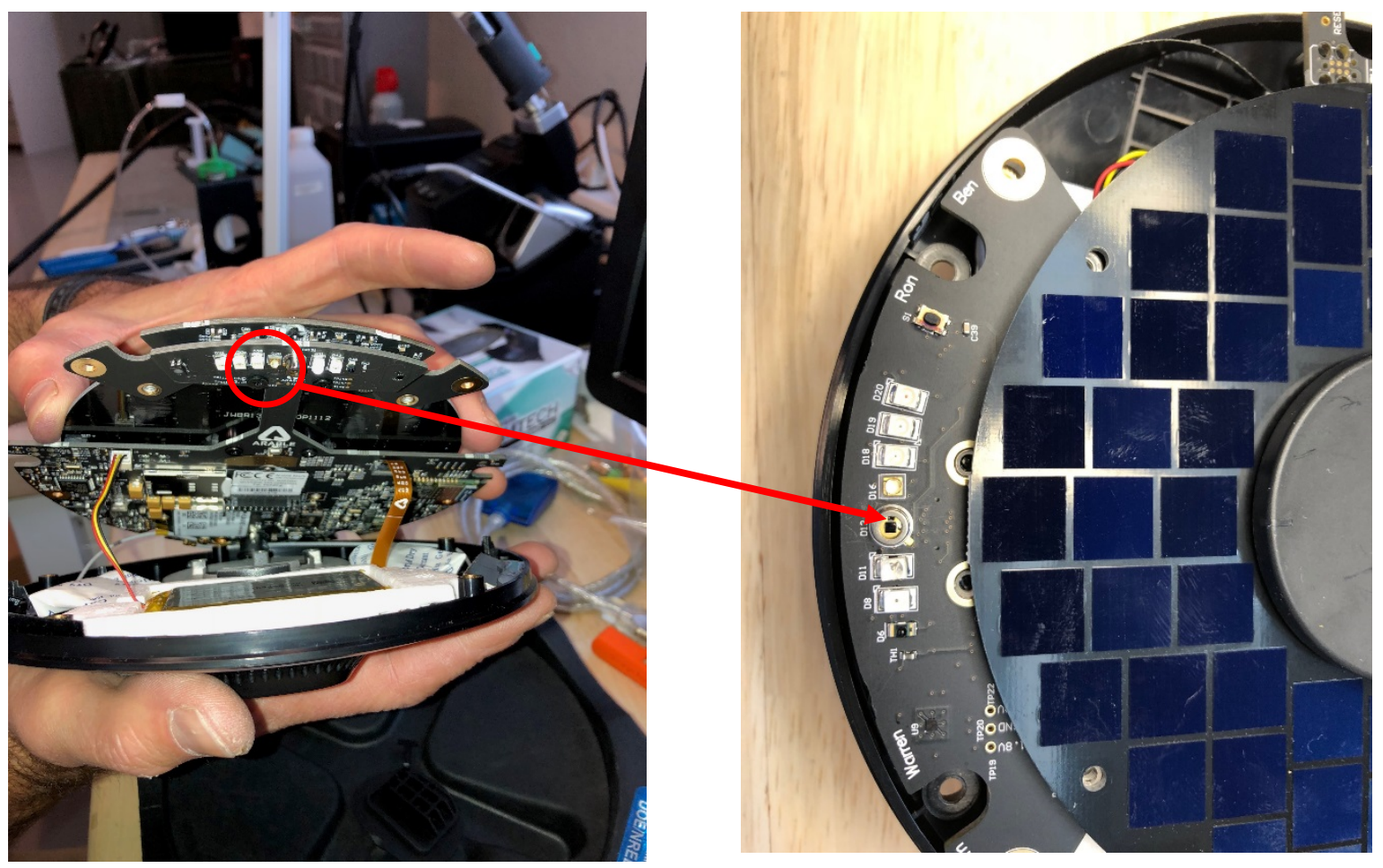

Figure 17. NREL upgraded the shortwave silicon photodiode sensor in the device from a spectral irradiance wavelength of 400-700 nm to 400-1100 nm. Photos by Peter Gotseff, NREL

The new sensor is a Hamamatsu Photonics model with good operating temperature range ($\left.40^{\circ} \mathrm{C}-100^{\circ} \mathrm{C}\right)$. Further, the sensor has good temperature response, although the temperature 
response deviation increases above $1050 \mathrm{~nm} .{ }^{1}$ This deviation is typical for silicon photodiode sensors.

\subsection{New External Leveling Apparatus}

Currently, Arable's Mark device is equipped with an internal leveling system that can be accessed using the button in the device, and the four light-emitting diode lights will beam in blue if the device is leveled. Then the leveling and orientation data can be retrieved through cloud communication. However, NREL designed a mounting pole with an attached bubble level to aid with ease of installation and routine maintenance and operation. The pole was fabricated in the NREL machine shop. Figure 18 shows the design of the new apparatus, which includes a connector to the base of Arable's Mark device and an extended bubble level. This feature is essential because measurement accuracy depends on Arable's Mark device being level. Over time, the device orientation could change because of wind, operator error, or other reasons, which causes the device to become unlevel and triggers data inaccuracy. Therefore, having this external leveling apparatus can be part of routine maintenance, which will assist in reducing data inaccuracy.
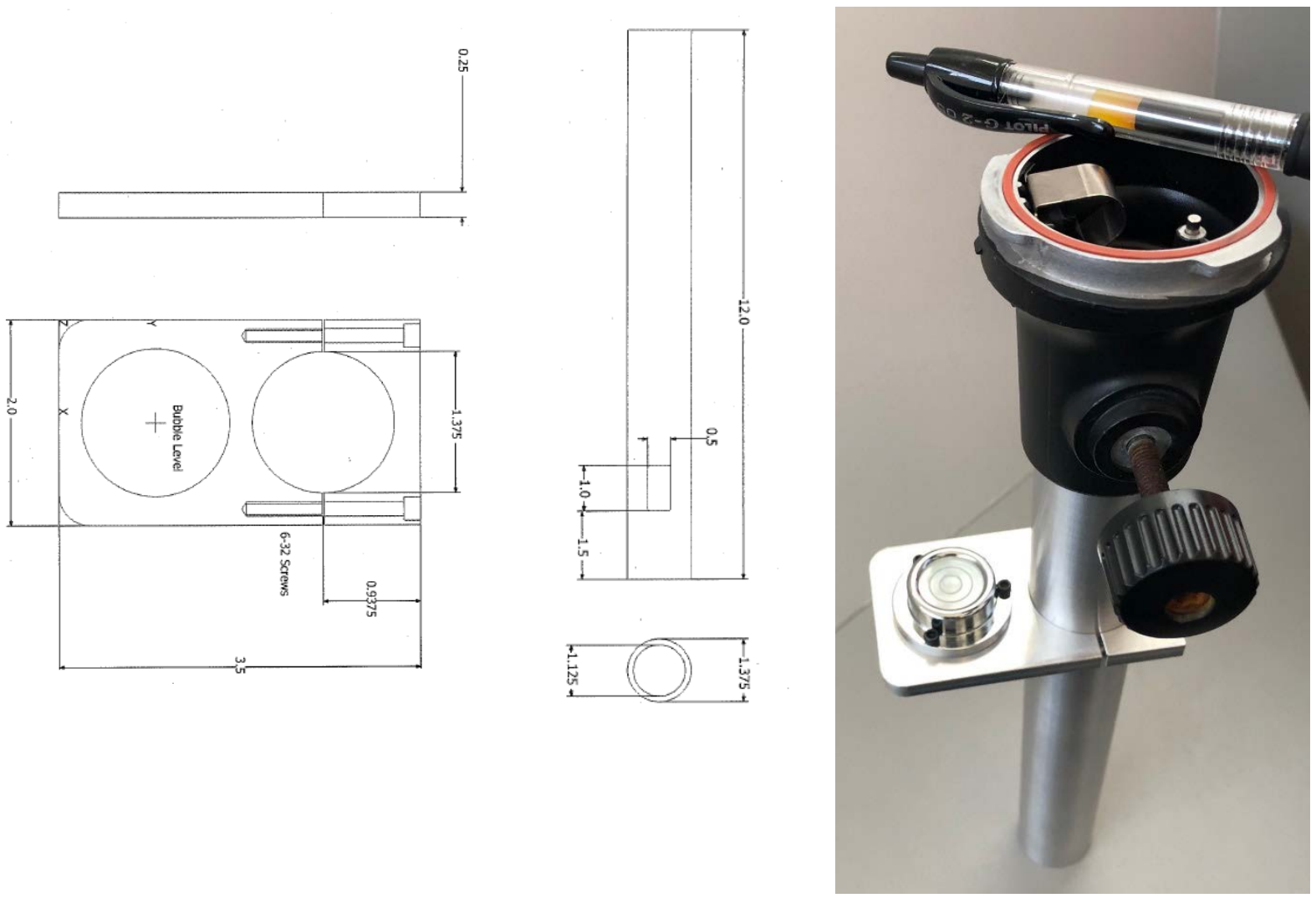

Figure 18. Design and image of Arable's new Mark leveling apparatus. Photo by Afshin Andreas (Left; design) and Aron Habte (right), NREL

\footnotetext{
${ }^{1}$ See https://www.hamamatsu.com/us/en/product/type/S1336-5BK/index.html.
} 


\section{Device Deployment for Solar Energy Applications}

NREL and Hawaiian Electric deployed three Arable Mark sensors at PV installations on Oahu Island in Hawaii. The sensors were installed adjacent to existing rooftop PV arrays for the purpose of estimating maximum available PV power at times when the PV output was curtailed. The sensors were selected because they are the only known self-contained, self-powered, off-theshelf solution for remote solar irradiance sensing and data collection. Other typical solutions would require integrating several separate components (power supply, battery, sensor, data recording equipment, and communications equipment). It was known at the time of installation that the solar irradiance data collected by the Arable units are intended for agricultural applications, so it was anticipated that the data would need adjustments for use in PV plant production validation. This limitation would likely preclude the use of the Arable sensors for PV applications outside very limited research settings; however, it would likely be simple to modify the Arable devices to include a PV reference cell or other global irradiance sensor appropriate for PV applications. It would also be important to provide a way to ensure that the irradiance sensor is oriented in a specific direction (typically in the plane of the PV array), which is currently somewhat difficult given the slightly dome-shaped form of the top surface. It is not clear how large the market might be for such devices, but the market is expected to grow as PV systems proliferate and as interest in validating PV production (and estimating possible PV production) grows.

The Arable devices performed well in the field - they were relatively easy to deploy and track their status. Accessing the data via the application programming interface was straightforward. The data proved to be useful but required significant nontrivial post-processing to obtain useful data for PV production estimation.

The calibration values from the manufacturer were used for these devices; however, similar cosine and tilt corrections were carried out using Eq. 3 to Eq. 5. The offset values in Eq. 4 were selected by trial and error. The AC reading from the PV panels power output from the locations assisted in selecting an optimum offset value. Thus, the offset values were manually optimized until a linear relationship between $\mathrm{AC}$ and arable irradiance was improved (Figure 19, right).
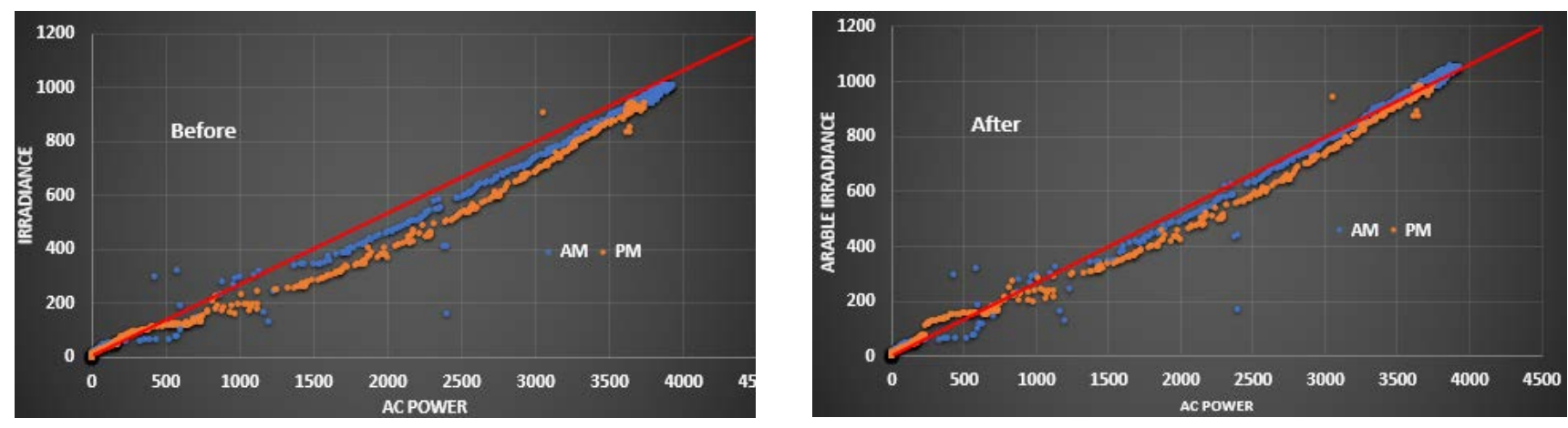

Figure 19. Before applying Eq. 3 (left) and after applying the correction equation (right)

After applying the correction, the result showed a good linear relationship (Figure 19, right). Figure 20 shows the comparison among the various measurements where Arable's Mark device was deployed for June 2 (top). The bottom panel shows a magnified view of a few days under all 
sky conditions (June 12-June 17). As shown in the figure, the characterization assisted in improving the irradiance data from Arable's Mark device. The improvement is because of the application of the cosine and tilt correction methodology, and thus after correction the uncorrected irradiance values changed to new corrected values, as shown in the figures.

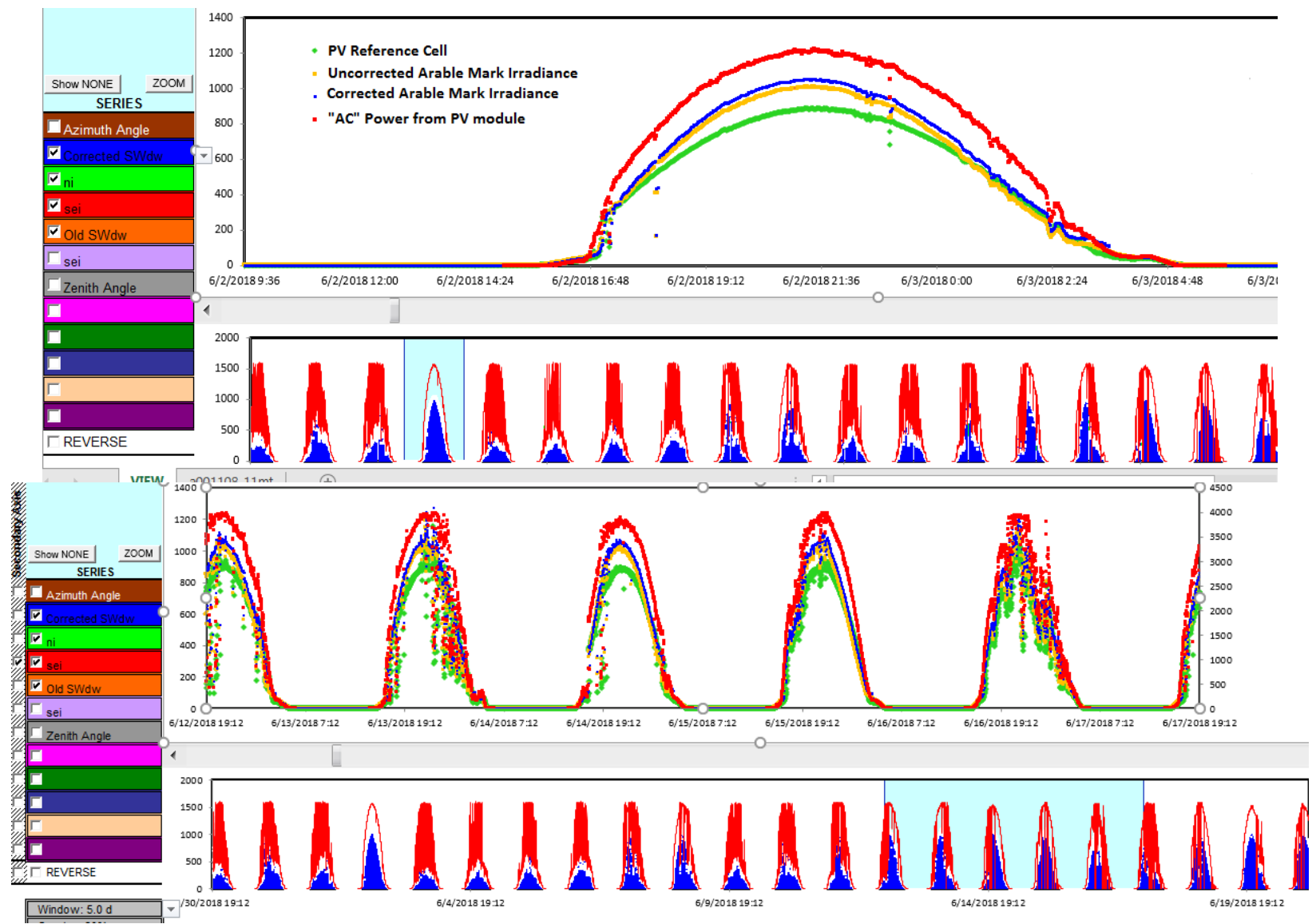

Figure 20. Comparison of measurement: red is AC power from the PV module, blue is the corrected Arable irradiance, orange is the uncorrected Arable irradiance, and green is the PV reference cell. The top panel shows a magnified view for a clear day, and the bottom panel shows a few days under all sky conditions. 


\section{Challenges}

Arable's Mark device provides integrated analytical solutions for agricultural problems. The device is equipped with multiparameter and low-cost sensors; however, the device requires some changes for use in solar energy projects. Some of the challenges are as follows:

- Ensuring and determining the long-term stability of Arable's Mark device is essential. For example, Arable's Mark diffuser changes color through time, which we suspect might create attenuation of the signal through time (Figure 21). Therefore, we recommend performing calibration of the device at least every year. The device could be sent back to the manufacturer for recalibration, or an in situ comparison with reference radiometers could be performed. Further, Arable should explore replacing the current diffuser, which is prone to discoloration, with another type of diffuser, e.g., an acrylic diffuser.

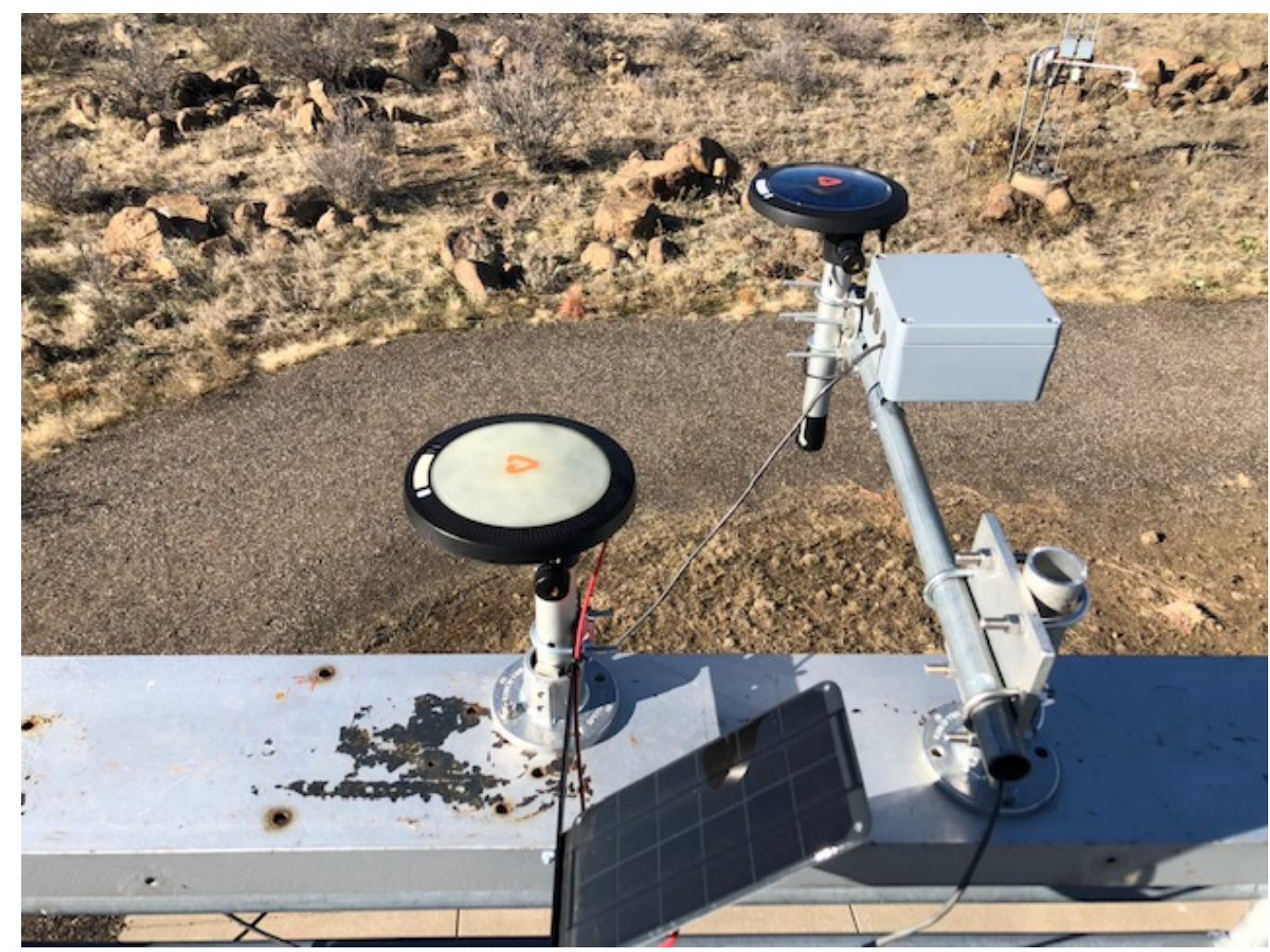

Figure 21. Discoloring of Arable's Mark top surface (left) compared to a newly deployed Arable Mark (right), Photo by Aron Habte, NREL

- For some solar energy applications, such as PV plant operators, having a direct connection to a data logger or existing data acquisition system from Arable's Mark device would be essential because having the solar resource data and other meteorological parameters in the same data stream with the power data will assist in understanding and responding to the temporal variability of solar radiation. This is important to optimally integrate the power output of PV systems onto electric grids.

- There seem to be some remaining bugs in the Arable devices that are causing them to occasionally stop working for unknown reasons, resulting in data loss. Ideally, it would 
be helpful if the devices could be remotely reset when needed, without requiring a user to manually reset them on-site. To some extent, some of the problems could be solved remotely.

- Performing factory upgrade of the shortwave sensor from 400-700 nm to 400-1100 nm is essential. Such a modification would make the sensors useful as a self-contained, selfpowered solution for gathering PV irradiance data from any location with cellular service.

- Providing specification or uncertainty levels for each sensor is essential for solar energy project phases. For these projects, requirements for the measurement accuracy and transparency are needed; otherwise, the economic consequences of unanticipated errors are catastrophic. 


\section{Summary and Future Work}

NREL, in collaboration with Arable Labs Inc., worked to improve Arable's Mark device instrumentation and measurement systems available for measuring and monitoring solar radiation elements needed by electric utilities and solar power system integrators. Arable's lowcost system contains a combination of fast-response detectors that provide meteorological and trending information, which are essential for solar resource assessments and forecasting in solar energy projects. The proposed work used NREL's SRRL, which is an International Organization for Standardization 17025 accredited calibration facility, and expertise to perform accurate spectral and broadband calibration and characterization of the fast-response detectors in Arable's system and assist Arable in developing a published methodology that will enable implementation of the results into their production pipeline, resulting in more accurate solar resource measurements for end-user solar energy organizations. The methodology for calibration and characterization reported here demonstrated promising results for Arable's Mark device compared to existing photodiode pyranometers, such as the LI-200 sensor from LICOR. The calibration and characterization methodologies employed by NREL resulted in a small bias compared to the reference GHI data obtained with a thermopile pyranometer and a small bias compared to the three conventional photodiode sensors. After recalibration, the spectrometer readings of the Mark sensors were found to be satisfactory compared to a reference spectroradiometer, at least under clear-sky conditions. More research is needed to evaluate the spectral accuracy under partly cloudy and rapidly changing conditions. The present calibration and characterization results, however, apply only to the UUT, location, and data period discussed here. It is too early to evaluate the instrument's uncertainty, and it is not possible to infer a broad conclusion based on the results of only the specimens tested here at NREL.

Both indoor and outdoor calibration methods for both spectral and broadband irradiances are applicable to the Arable device. The indoor calibration can be carried out by calibrating Arable's Mark devices using a NIST traceable lamp and the outdoor calibration by using a reference broadband radiometer, such as an EKO Instruments model MS-80.

As discussed in Section 4, the J-Link interface supplied by Arable has the USB interface. NREL might purchase a J-Link with an Ethernet connection in hopes of improving the mounting and communications options. By using an Ethernet connection, the location of the Mark and the computer are not limited by USB cabling. In addition, the USB driver is no longer required; however, the J-Link commander software might still be required. If the J-Link commander were part of the J-Link device, this would allow for reliable data collection through a Campbell data logger, which is commonly used for resource assessment projects at NREL.

When the Mark is mounted outdoors, care should be taken to ensure that the instrument is level. Even though Arable's Mark contains light-emitting diodes that inform the user if the device is level, the user must press the main button and four lights will beam in blue if the device is level. It is hard to see the lights in the field, however; therefore, NREL designed a mounting pole with an attached bubble level to aid in mounting. The pole is fabricated in the NREL machine shop and installed by SRRL personnel.

In the future, NREL will continue to work with Arable through a cooperative research and development agreement or other means to assist in developing the relationships needed to deploy 
improved Arable's Mark devices. These efforts, if successful, could ensure that these sensors reach solar energy end users, where they could have a significant impact. 


\section{References}

1. Manajit Sengupta, Aron Habte, Christian Gueymard, Stefan Wilbert, Dave Renné, and Thomas Stoffel, Best Practices Handbook for the Collection and Use of Solar Resource Data for Solar Energy Applications: Second Edition (NREL/ TP-5D00-68886) (Golden, CO: National Renewable Energy Laboratory, 2017). https://www.nrel.gov/docs/fy18osti/68886.pdf.

2. Aron Habte, Manajit Sengupta, Afshin M. Andreas, Ranganath Narasappa, Taylor Thomas, Adam Wolf, and Christian A. Gueymard, "Characterization of a Low-Cost Multi-Parameter Sensor for Resource Applications: Preprint" (Presented at the 2018 World Conference on Photovoltaic Energy Conversion (WCPEC-7), Waikoloa, Hawaii, June 10-15, 2018). https://www.nrel.gov/docs/fy18osti/71585.pdf.

3. Aron Habte, Manajit Sengupta, Afshin Andreas, Stephen Wilcox, and Thomas Stoffel, "Intercomparison of 51 Radiometers for Determining Global Horizontal Irradiance and Direct Normal Irradiance Measurements," Solar Energy 133 (2016): 372-393. https://doi.org/10.1016/j.solener.2016.03.065.

4. T. Landelius and W. Josefsson, "Methods for Cosine Correction of Broadband UV Data and Their Effect on the Relation Between UV Radiation and Cloudiness," Journal of Geophysical Research: Atmospheres.

5. Gunther Seckmeyer and Germar Bernhard, "Cosine Error Correction of Spectral UVIrradiances," Proceedings of SPIE 2049, Atmospheric Radiation (November 2, 1993). doi: 10.1117/12.163505; https://doi.org/10.1117/12.163505. 